\title{
Attentional brain rhythms during prolonged cognitive activity
}

5 1. Institut des Sciences Cognitives Marc Jeannerod, CNRS UMR 5229, Université Claude Bernard Lyon 1,

667 Boulevard Pinel, 69675 Bron Cedex, France

7

8 Corresponding author: Corentin Gaillard, corentin.gaillard@isc.cnrs.fr, Carine De Sousa,

9 carine.desousa@isc.cnrs.fr, Suliann Ben Hamed, benhamed@isc.cnrs.fr

* These two authors contributed equally to this work

Running title: Rhythms of prolonged sustained attention

13 Keywords: sustained attention, perception, cognitive demand, prolonged mental effort, prefrontal 


\section{Abstract (250):}

17 As routine and lower demand cognitive tasks are taken over by automated assistive systems, human 18 operators are increasingly required to sustain cognitive demand over long periods of time. This has been 19 reported to have long term adverse effects on cardiovascular and mental health. However, it remains 20 unclear whether prolonged cognitive activity results in a monotonic decrease in the efficiency of the 21 recruited brain processes, or whether the brain is able to sustain functions over time spans of one hour 22 and more. Here, we show that during working sessions of one hour or more, contrary to the prediction 23 of a monotonic decline, behavioral performance in both humans and non-human primates consistently 24 fluctuates between periods of optimal and suboptimal performance at a very slow rhythm of circa 5 cycles per hour. These fluctuations are observed in both high attentional (in non-human primates) and low attentional (in humans) demand conditions. They coincide with fluctuations in pupil diameter, indicating underlying changes in arousal and information-processing load. Accordingly, we show that these rhythmic behavioral fluctuations correlate, at the neurophysiological level, with fluctuations in the informational attention orientation and perception processing capacity of prefrontal neuronal populations. We further identify specific markers of these fluctuations in LFP power, LFP coherence and spike-field coherence, pointing towards long-range rhythmic modulatory inputs to the prefrontal cortex rather than a local prefrontal origin. These results shed light on the resilience of brain mechanisms to sustained effort and have direct implications on how to optimize high cognitive demand working and learning environments. 


\section{Introduction}

38

39

40

41

42

43

44

45

46

47

48

49

50

51

52

53

54

55

56

57

58

59

60

61

62

63

64

65

66

67

68

69

A six hours long university exam or a routine maneuver at the international space station require highly sustained performance and any drop in cognition can have dramatic outcomes. We are increasingly faced with such situations in which memory and decision-making processes need to be sustained at high performance for long time periods. However, maintaining cognitive processes at an optimal regime comes at a cost. For example, prolonged activity has long term adverse effects on health, ranging from a higher rate of cardiovascular diseases to fatigue, reduced sleep duration and depression(van der Hulst, 2003; Johnson and Lipscomb, 2006; Liu and Tanaka, 2002; Sekine et al., 2006; Shields, 1999; Sokejima and Kagamimori, 1998). In addition, prolonged cognitive activity also results in a decrease in cognitive performance associated with undesirable decision-making, distress and burnout(Lockley et al., 2004; Proctor et al., 1996; Virtanen et al., 2009). In particular, sustained attention over long time periods has been linked to mental fatigue and cognitive exhaustion(Bonnefond et al., 2010; Guo et al., 2016; Warm et al., 2008).

The short term influence of motivational factors such as reward history(Abrahamyan et al., 2016; Urai et al., 2019), or error history(Cowley et al., 2020; Pisupati et al.; Weissman et al., 2006), on decisions and neuronal processes have been extensively studied and modelled. In contrast, the long-term influence of task-independent factors such as fatigue(Marcora et al., 2009), arousal(Aston-Jones and Cohen, 2005; McGinley et al., 2015; Urai et al., 2019), and satiety(Allen et al., 2019), are less understood. In particular, the neuronal mechanisms associated with sustained cognitive performance over prolonged periods of time are to date poorly understood and it remains unclear whether prolonged cognitive activity results in a monotonic decrease in the efficiency of the recruited brain processes, or whether the brain is actually able to sustain functions over time spans of one hour and more. A recent study in non-human primates reports that, during prolonged cognitive tasks, a slow drift in the neuronal activity of the visual and prefrontal cortex is associated with enhanced impulsive decisions(Cowley et al., 2020).

In the following, we performed a cross-species study in which we show that during working sessions of one hour or more, behavioral performance in both humans and non-human primates, consistently fluctuates at a very slow rhythm of the order of 5 cycles per hour. Importantly, we show that these behavioral fluctuations are qualitatively similar in both species (humans and macaques), during the performance of tasks involving different levels of attentional engagement. We characterize the neurophysiological correlates of these ultra-slow behavioral fluctuations by analyzing variations in pupil size in humans and variations in prefrontal perceptual and attention neuronal processes in non-human primates. 
Specifically, we trained two macaque monkeys to perform a very long sustained attention task lasting from one to four hours. We simultaneously recorded multi-unit neuronal activity (MUA) and local field potentials (LFP), from multiple recording sites in the frontal eye field (FEF), a prefrontal cortical area at the source of spatial attention control signals(Astrand et al., 2016; Buschman and Miller, 2007a; Ekstrom et al., 2008; Gregoriou et al., 2009a; Ibos et al., 2013). We report large fluctuations in attentional behavioral performance, by up to 10\%, at an ultra-slow rhythm of 4 to 7 cycles per hour (i.e. every 9 to 15 minutes). Applying machine learning approaches to decode prefrontal attentional related information(Astrand et al., 2016, 2020; De Sousa et al., 2021; Gaillard et al., 2020a), we show that these fluctuations in behavioral performance coincide with phase-locked rhythmic fluctuations in how well the prefrontal cortex both accurately encodes the perception of incoming visual stimuli and spatial attention orientation information. In addition, we show that high behavioral performance task epochs coincide with the enhanced of multiple neurophysiological attentional markers: enhanced theta $(4-6 \mathrm{~Hz})$ and beta $(18-24 \mathrm{~Hz})$ LFP power, enhanced alpha $(7-11 \mathrm{~Hz})$ LFP coherence, enhanced spike-field coherence in the theta $(4-6 \mathrm{~Hz})$, alpha $(7-11 \mathrm{~Hz})$ and gamma $(35-50 \mathrm{~Hz})$ frequencies. In humans, we further show that these fluctuations in behavioral performance coincide with rhythmic variations in pupil size, pointing towards underlying changes in arousal and information-processing load. Overall, this suggest that the hour-scale variations in cognitive performance arise from long range mechanisms, impacting local neuronal spiking activity through selective changes in spike-field coherence. The net effect is an overall variation in the efficiency of brain attention and perceptual related processes. These results are discussed in the context of perceptual and attentional rhythms and the resilience of brain mechanisms to sustained effort. In addition to shedding light on how the brain functions under prolonged cognitive activity, this work has direct implications on how to optimize high cognitive demand working and learning environments to reconcile short term effort with both long-term performance and mental health.

\section{Results}

In order to describe modulations of attentional behavioral performance, and their prefrontal neuronal correlates, we had monkeys and humans perform a cognitive task for very long durations while respectively either recoding prefrontal neuronal activity or pupil size. Specifically, we had two monkeys perform a demanding attentional task for one to four hours, depending on the motivation of the monkeys. Monkeys were required to perform a cued target-detection task (Fig. 1a) while we recorded the MUA and LFP activity bilaterally the frontal eye fields (FEF), using two 24-contacts recording probes (Fig. 1b). Target luminosity was adjusted so as to enhance the attentional relevance of the cue, and distractors were introduced in order to force the monkeys to only respond to the target. Fig. 1c shows 

duration was 3.94 hours for monkey M1 (s.e. $=0.66$ ) and 2.99 hours for monkey M2 (s.e. =0.20). Overall, behavioral performance did not vary between the beginning (defined as the initial 20 minutes of the task) and the end of the behavioral session (defined as the last 20 minutes of the task, Wilcoxon nonparametric test, $p=0.40$ ), which indicates that we did not find a trend of decreasing performance along the session. Human subjects (35 subjects in all), had to detect a shivering Gabor in the midst of slowly drifting Gabors of variable orientations. Task duration in human subjects was fixed to $75 \mathrm{~min}$. As in macaques, there was no significant changes in median reaction times when comparing the first versus the last 20 minutes of the task (Wilcoxon non-parametric test, $p=0.95$ ).
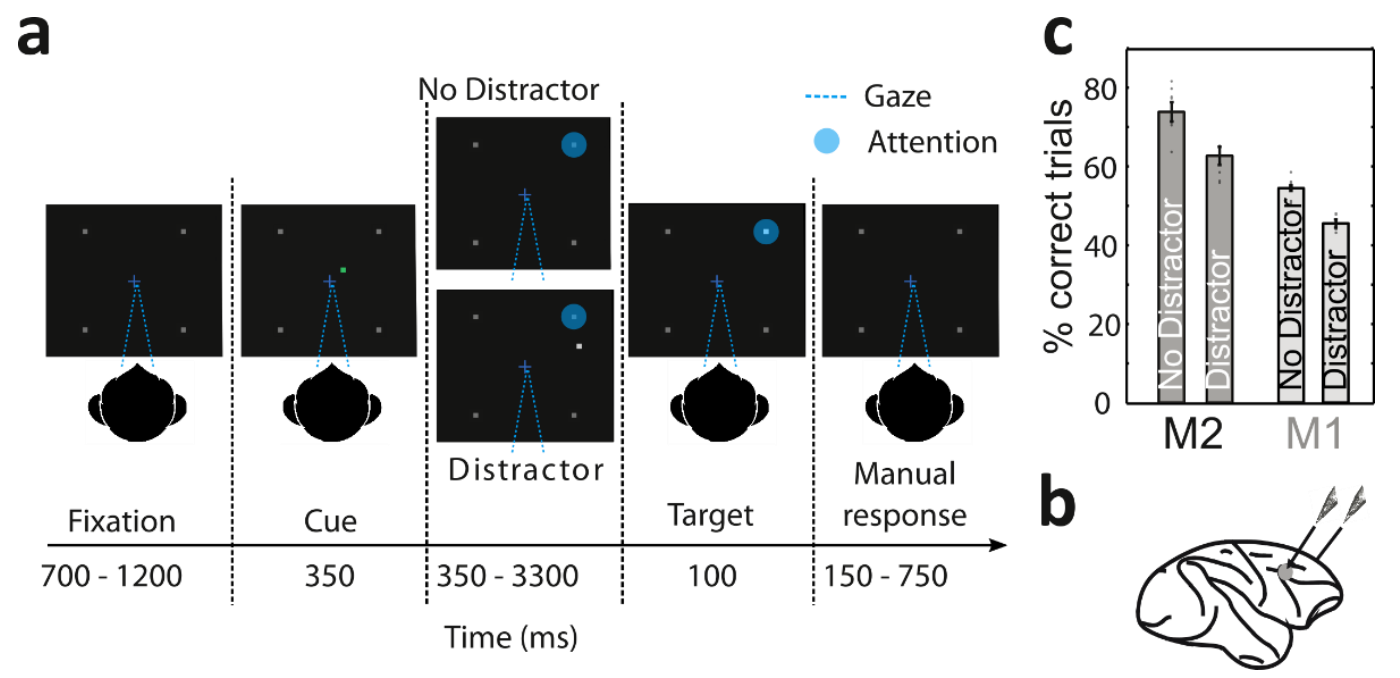

Figure 1: Task design and behavioral performance. (A) 100\% validity cued target detection task with temporal distractors. Monkeys had to hold a bar and fixate a central cross on the screen for a trial to be initiated. The monkeys received a liquid reward for releasing the bar 150 - 750 ms after target presentation. Target location was indicated by a cue (green square, second screen). Monkeys had to ignore any uncued event. (B) Recording sites. On each session, 24-contacts recording probes were placed in each FEF. (C) Behavioral performance of monkeys $\mathrm{M} 1$ and $\mathrm{M} 2$ at detecting the target in the presence or absence of a distractor (median \% correct +/- median absolute deviations, dot correspond to individual sessions).

\section{Behavioral performance varies rhythmically at 4 to 7 cycles per hour}

Hit and miss trials were sorted according to their respective time in the session and a running estimate of monkeys' behavioral performance was estimated across the whole session time (see methods for detailed behavioral performance estimation procedures). Fig. 2a represents the running estimate of behavioral performance in an exemplar session, defined by the ratio between hit trials and the sum of hit and miss trials. In this figure, we observed a clear periodic fluctuation pattern of the behavioral 
127 performance by up to $30 \%$ along the entire session. The spectral density decomposition of this

128 behavioral performance time series as estimated using wavelet transforms exhibits an oscillatory peak 129 at 5.7 cycles per hour (Fig. $2 \mathrm{~g}$, gray, permutation test, $\mathrm{p}<0.05$ ). Such rhythmic modulations of behavioral performance can be described consistently in all recording sessions and both subjects, at an average frequency of 5.59+- 0.30 cycles per hour (Fig. 2h, gray). Over all sessions, behavioral performance was, on average, $16 \%$ higher at the optimal phase (i.e. the phase maximizing behavioral performance) of each cycle of the behavioral oscillation compared to the anti-optimal behavioral phase (i.e. the phase

a

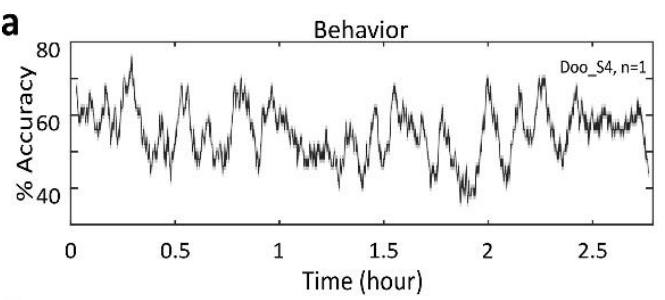

C

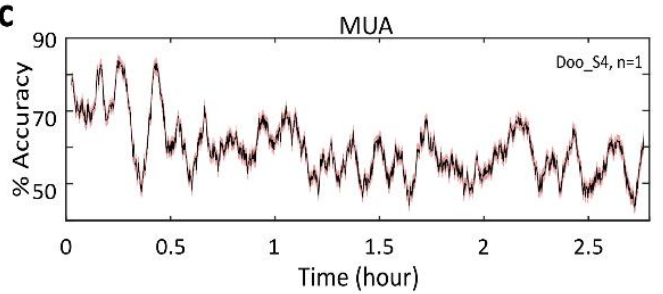

e

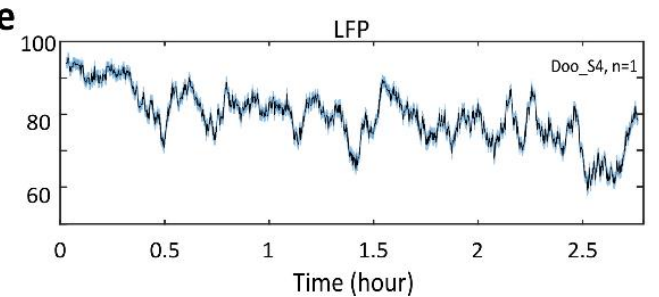

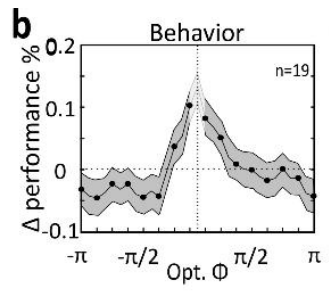

g
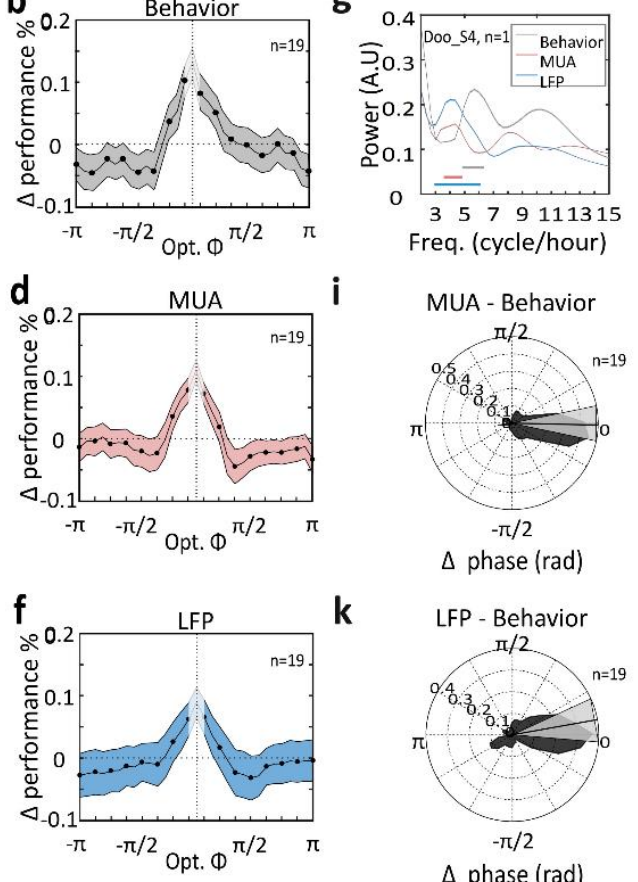

$\Delta$ phase (rad)

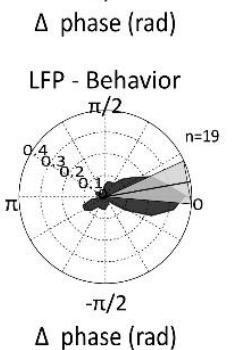

$\Delta$ phase $(\mathrm{rad})$

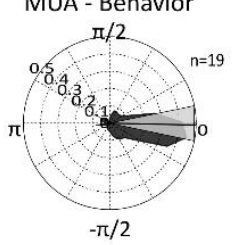

h

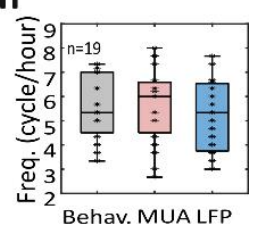

j

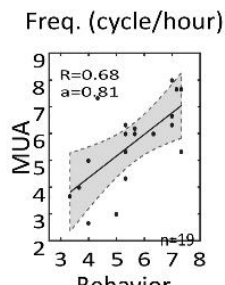

I Freq. (cycle/hour)

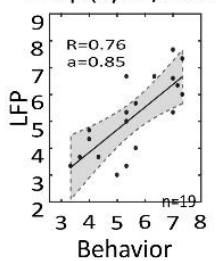
hit vs. miss trials from prefrontal MUA and LFP signals. (A) Running behavioral performance (Hits/(Hits+Misses)) estimates throughout session time (mean +/- s.e.) for the exemplar session Doo_S4. (B) Variations in overall behavioral performance across all sessions, as a function of phase difference from optimal phase. (C) Running estimates of target encoding classification accuracies for hit vs. miss classification from prefrontal MUA frequency content, throughout session time (mean +/- s.e.), for the same exemplar session as in (A). all sessions, as a function of phase difference from optimal phase. (E) Running estimates of target encoding classification accuracies for hit vs. miss classification from prefrontal LFP frequency content, throughout session time (mean + /- s.e.), for the same exemplar session 

frequency content across all sessions, as a function of phase difference from optimal phase.

(G) Frequency power spectrum of the time series presented in ( $A$, grey), ( $C$, pink) and ( $E$, blue). Epochs of statistical significance against the $95 \%$ C.I. indicated by lines of same color. +/- s.e.), for behavioral performance (grey) and hit vs. miss decoding accuracies from prefrontal MUA (pink) and LFP (blue), all identified peak frequencies were significant against 95\% C.I (see method). (I-K) Phase difference and (J-L) correlation between ultra-slow oscillations identified in behavioral performance and MUA or LFP hit vs. miss decoding accuracies.

Previous studies indicate that variations in behavioral performance might be interpreted as fluctuations in how accurately subjects encode the task-relevant visual information(Busch et al., 2009; Jasper et al., 2019; Parto Dezfouli et al., 2018). Accordingly, an enhanced (respectively impaired) prefrontal representation of visual stimuli is expected during the phase of optimal (respectively poor) behavioral performances (van Vugt et al., 2018). In order to test this hypothesis, we apply machine learning procedures(Astrand et al., 2016, 2020; Gaillard et al., 2020a) to ongoing LFP/MUA activities following target presentation to decode behavioral outcome (Hit or Miss) based on FEF neuronal response to target presentation. Specifically, we analyze how much the ultra-slow oscillations detected in behavioral performances impact correct (Hit) or incorrect (Miss) response prediction based on FEF neuronal population responses to the target (See methods for more details about decoding procedures).

Figure $2 \mathrm{c}$ represents, for the same session as presented in figure $2 \mathrm{a}$, the running estimates of decoding accuracies for hit vs. miss classification from prefrontal MUA frequency content, throughout session time. We observe that decoding accuracy varies in time by up to $34 \%$ on this exemplar session (Fig. 2c).

171 A clear rhythmic fluctuation of decoding accuracies can be identified, peaking at 4.74 cycles per hour 172 (Fig. 2g, pink, permutation test, $\mathrm{p}<0.05$ ), closely matching the frequency identified in the behavioral 173 performance (5.7 cycles per hour). Rhythmic modulation of decoding accuracies can be described 174 consistently in all recording sessions from both subjects at an average frequency of $5.67+-0.27$ cycles per hour (Fig. 2h, pink). Over all recording sessions, decoding accuracies are on average over $13 \%$ higher at the optimal behavioral phase (i.e. the phase maximizing decoding accuracy) as compared to the antioptimal behavioral phase (i.e. the phase minimizing decoding accuracy, Fig. 2d). Similarly, we observe rhythmic modulations of LFP based behavioral classification (Fig. 2e, blue) identified for this exemplar session at a specific rhythm of 4.6 cycles per hour. This rhythm was identified in all sessions at an average 
181 accuracies were, on average, over $11 \%$ higher at the optimal behavioral phase as compared to the anti-

182 optimal behavioral phase (Fig. 2f).

183 Crucially, across all recording sessions, peak oscillatory frequencies identified in the behavioral 184 performance and in the MUA decoding accuracy of hits vs. misses are highly correlated (Fig. 2j, Pearson 185 correlation, $\left.r^{2}=0.68, p<0.001\right)$. This is remarkable given the fact that at these time scales, any source of 186 noise is expected to have a strong impact on the identification of peak frequency from any of the three 187 considered time series. In addition, a clear phase locking between these two rhythms can be observed 188 between the two signals (Fig. $2 \mathrm{i}$, circular Kuiper's test for equal distributions, $p<0.05$ ), confirming that 189 the identified periods of low (resp. high) subject behavioral performance coincide with decreased (resp. increased) prefrontal accuracy of visual stimuli coding. Similarly, the frequency peaks of the LFP based 191 target decoding accuracies highly correlate with the behavioral oscillatory peaks (Fig. 2i, Pearson correlation, $r^{2}=0.76, p<0.001$ ), and are highly significantly phase locked (Fig. 2k, circular Kuiper's test for equal distributions, $p<0.05)$. Complementing these data, the peak frequency of the LFP based target decoding accuracies highly correlate with the MUA based target decoding accuracies oscillatory peaks

195 (Supplementary Fig. 2, Pearson correlation, $r^{2}=0.63, p<0.01$ ), and are significantly phase locked 196 (Supplementary Fig. 2, circular Kuiper's test for equal distributions, $p<0.05$ ).

197 Overall, these results indicate a functional link between the observed change in discriminability between 198 hits and misses from the MUA/LFP signals and behavior. Behavioral performance on this specific 199 attentional task is dependent of the correct allocation of attentional processes (Bello et al., 2020; 200 Posner, 2016). As a result, the ultra-slow fluctuations in target processing and perception is expected to 201 directly impact FEF attentional-related information(Astrand et al., 2016; Gaillard et al., 2020a) to the 202 same extent.

\section{Prefrontal MUA based decoded attentional information varies rhythmically at 4 to 7 cycles per hour}

Prefrontal population attention related information, can be assessed by machine learning techniques applied to ongoing prefrontal population multiunit (MUA) neuronal ensemble activity(Astrand et al., 2016; De Sousa et al., 2021; Gaillard et al., 2020a). In the following, we show that prefrontal population attention-related information is modulated at an ultra-slow rhythm that correlates with the rhythmic variations in behavioral performance described above. Specifically, we focus on correct trials, i.e. on trials in which spatial attention was (a priori) correctly oriented to the expected target location. On each

210 correct trial, we compute the locus of the attentional spotlight just prior to target presentation and we 211 test whether attention was properly oriented in the cued visual quadrant or not. We then compute a 212 running average of attention decoding accuracy throughout the time of the session (Fig. 3a, same 
exemplar session as in figure $2 \mathrm{a}$ and $2 \mathrm{c}$ ). We observe that decoding accuracy varies in time by more than $20 \%$. In other words, how well attention-related information can be extracted from the prefrontal cortex population activity varies as a function of time in the session. Using wavelet transform, we identify for this specific session a peak in MUA attention-related information at 5.67 cycles per hour, matching the peak identified in the behavioral performance time series (Fig. 3e). Such rhythmic modulations in prefrontal population attention information can be described consistently in all recording sessions and both subjects, at an average frequency of $5.55+-0.23$ cycles per hour (Fig. 3f, magenta). On average, over all recording sessions, MUA-based attention related information estimates were over $10 \%$ higher at the optimal phase as compared to the anti-optimal phase (Fig. 3b). Crucially, ultra-slow rhythmic variations in prefrontal attention-related information are highly correlated with the

a

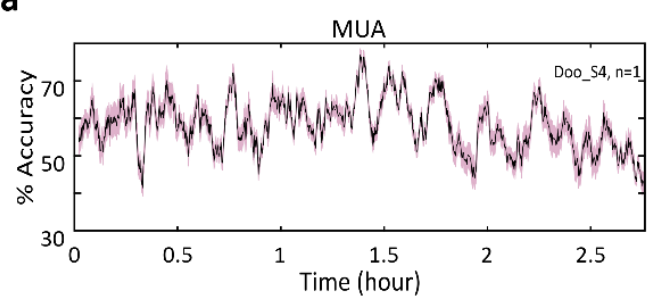

c

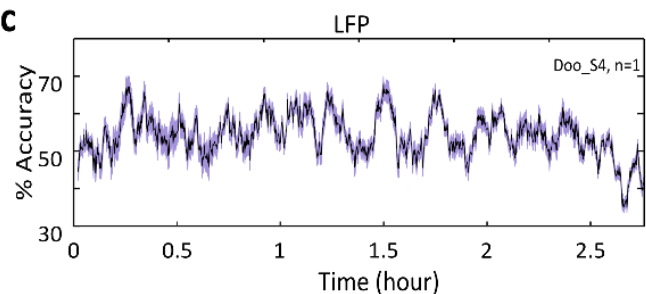

b
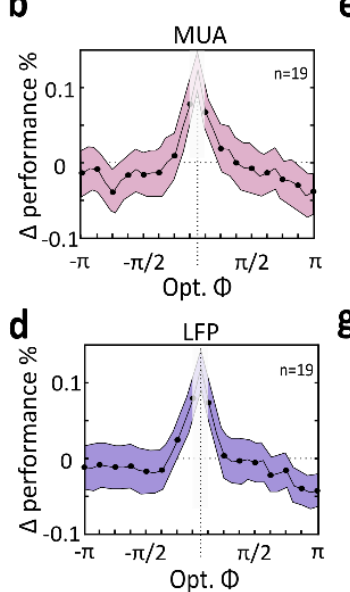

e

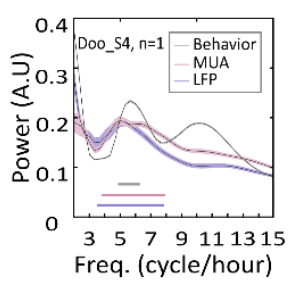

g

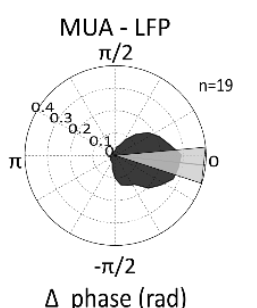

f

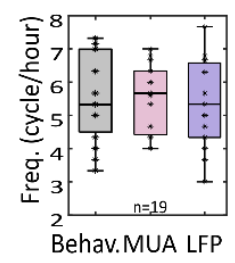

$\mathrm{h}$

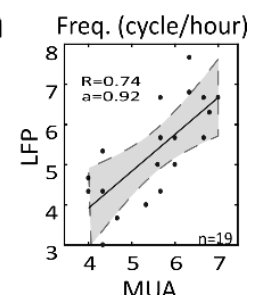

Figure 3: Ultra-slow rhythmic variations in prefrontal MUA and LFP attention-related information. (A) Running estimates of attention decoding accuracies from prefrontal MUA frequency content, throughout session time (mean +/- s.e.), for the exemplar session Doo_S4 (same as in figure 2). (B) Variations in overall MUA-based attention decoding accuracies across all sessions, as a function of phase difference from optimal phase. (C) Running estimates of attention decoding accuracies from prefrontal LFP frequency content, throughout session time (mean +/- s.e.), for the same exemplar session as in (A). (D) Variations in overall LFP-based attention decoding accuracies across all sessions, as a function of phase difference from optimal phase. (E) Frequency power spectrum of the time series presented in (A), (magenta) and (B), (purple). (F) Distribution of ultra-slow oscillatory peaks across all sessions and both subjects (median +/- s.e.), for MUA-based (magenta) and LFP-based (purple) attention decoding accuracies. (G) Phase difference and (H) correlation 
between ultra-slow oscillations identified in the MUA-based and LFP-based attention decoding accuracy time series.

240 Critically, subjects show more precise attentional orientation during the good phase of the ultra-slow

241 oscillation. Indeed, the rhythmic fluctuations in MUA attention-related information correspond to

242 variations in the distribution of the attentional spotlight prior to target presentation on the trials falling 243 on the peak (Fig. 4a) relative to those falling on the troughs of the ultra-slow rhythmic variations (Fig. 244 4b). Specifically, attention is more focused and closer to the expected target location by an average of $2452.1^{\circ}$, on peak trials relative to trough trials (difference in peaks of hit maps). The net effect of this is a covert attentional exploration of the cued location during the peak trials and a more distributed exploration of the screen on trough trials (Fig. 4c). These changes in prefrontal MUA attention-related information do not coincide with systematic changes in MUA average attention-related responses prior to target presentation. Indeed, there is no significant systematic variation in average MUA responses on peak trials relative to trough trials (Fig. S4 Wilcoxon rank sum test, $p>0.5$ ). This confirms that the

251 change in overall MUA attention-related information does not correspond to a non-specific mechanism 252 such as metabolic depletion, but rather to a rhythmic change in the prefrontal neuronal representation 253 of attentional processes.

\section{Prefrontal LFP based decoded attentional information varies rhythmically at 4 to 7 cycles per hour}

255 These variations in the population MUA attention-related information reflect changes in the population 256 neuronal code that subserves spatial attention in the prefrontal cortex. In order to test whether these 257 ultra-slow rhythmic fluctuations in the attentional code correspond to a local mechanism or whether 258 they reflect on more mesoscopic signals such as local field potentials (LFP), we reproduced the previous 259 analysis on these latter signals. Indeed, previous studies have demonstrated that very much like for 260 MUA signals, attention can be classified from the single trial LFP power content (mostly from the gamma 261 band), though to a lesser extent than from MUA signals(De Sousa et al., 2021; Tremblay et al., 2015). 262 On each correct trial, we compute the locus of the attentional spotlight just prior to target presentation 263 based on the recorded prefrontal LFP signals and we test whether attention was properly oriented in 264 the cued visual quadrant or not. We then compute a running average of attention decoding accuracy 265 throughout the time of the session (Fig. 3c, same exemplar session as in figure 2a, 2c, 2e and 3a). As 266 was seen for behavioral performance, hit/miss decoding from prefrontal LFPs and MUA-based attention 267 decoding, we observe that decoding accuracy varies in time by up to $20 \%$. Using wavelet transform, we identify for this specific session a peak in LFP spatial related information at $5 \mathrm{~Hz}$, matching the peak identified in the behavioral performance and MUA based attention information time series (Fig. 3e). 
recording sessions and both subjects, at an average frequency of $5.34+-0.29$ cycles per hour (Fig. 3f,

272 purple). On average, over all recording sessions, LFP-based attention related information estimates are 273 over $10 \%$ higher at the optimal phase as compared to away from this optimal phase (Fig. 3d). Crucially,

274 as is observed for MUA-based attention information, ultra-slow rhythmic variations in prefrontal 275 attention related information are correlated with the rhythmic variations in behavioral performance in 276 the same sessions (supplementary Fig. $3 b$, Pearson correlation, $r^{2}=0.58, p<0.05$ ). Interestingly, a significant correlation is observed across sessions between the oscillatory peaks of the MUA based attention related information and the LFP based attention related information. (Fig. $3 g, r^{2}=0.73$,

a

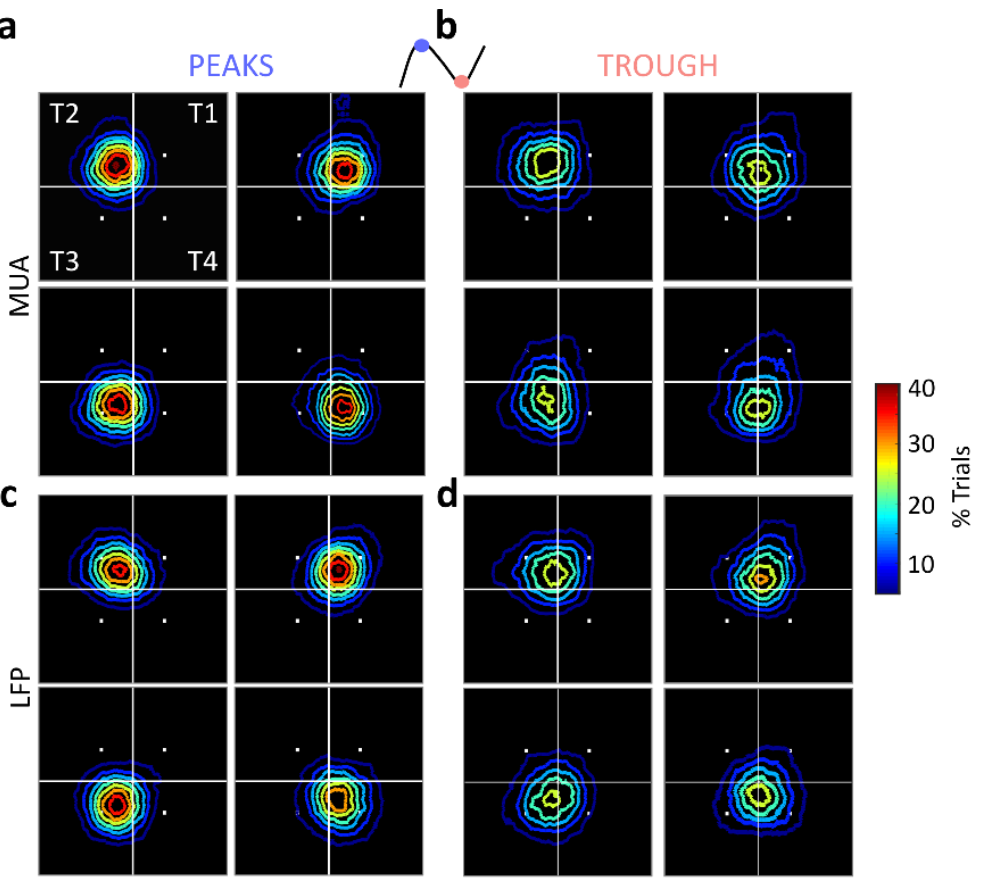

e

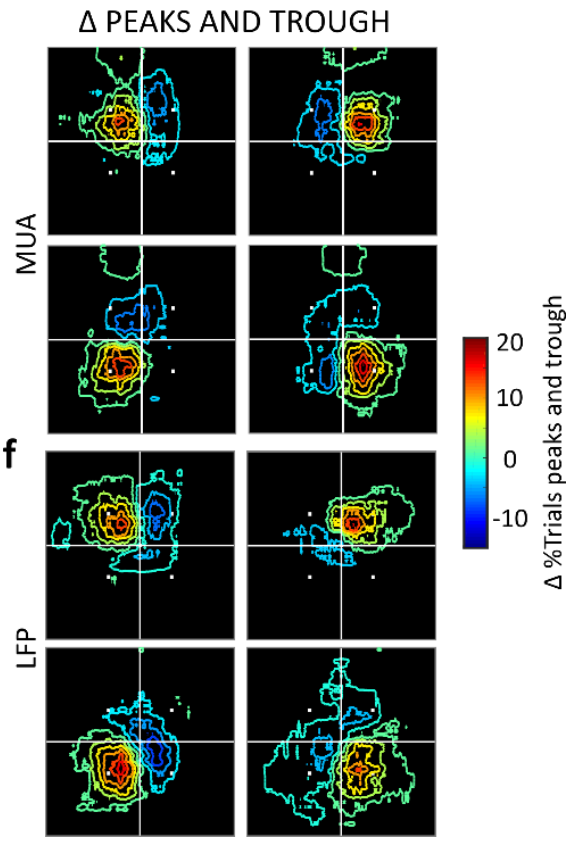
screen, the vertices of the central gray square indicate the locations where the cues are presented. The colored traces in panels A to B represent density (\% of trials) plots of where the spotlight falls on the screen, prior to target presentation, on correct trials, when the attention is cued to the upper left quadrant (upper left square), upper right (upper right square), lower right (lower right square) and lower left square (lower left square, see cartoon on the upper right). Panels $E$ and $F$ represent the difference between the maps presented in $A$ and $B$, and $C$ and $D$ respectively. 
293

As observed for the MUA-based attention-related information, the rhythmic variations in LFP attentionrelated information coincide with an over attentional exploration of the cued location during the peak trials (Fig. 4c) and a more distributed exploration of the screen on trough trials (Fig. $4 d$ and $4 f, 3.6^{\circ}$ average difference in peaks of hit map between the two conditions).

\section{MUA and LFP markers of the attentional ultra-slow oscillations}

Attention-related information modulation by ultra-slow oscillations could be observed from both LFP and MUA signals. This suggests that the observed slow oscillations might not reflect a local process but rather a more global mesoscopic process mediated by LFPs. In order to test this hypothesis, we computed prefrontal LFP power spectra during the $1000 \mathrm{~ms}$ attentional period preceding target presentation, on correct trials, using a continuous wavelet transform (see methods), and we segregated individual trial measures into trials falling in the peak or trough of the ultra-slow oscillations of the MUA attention-related information (see methods for more details). Average power spectrum differences across all sessions ( $n=19)$, all recording channels ( $n=48$ per session) and the two monkeys reveal a significant higher power in the peak relative to the trough of the oscillations, in two specific functional frequency bands (Fig. 5a, significance assessed against 95\% C.I.): a theta frequency band (mean= 6.35 $\mathrm{Hz}+-1.65$ s.e.), and a beta frequency band (mean= $24.97 \mathrm{~Hz}+-3.58$ s.e.). Importantly, theta and beta modulation of LFP activities have been associated with orientation of attention (Buschman and Miller, 2007b; Fiebelkorn et al., 2018a; Gaillard et al., 2020a; VanRullen, 2018). The observed modulation of these two frequency ranges during the ultra-slow oscillation cycles supports the idea that these oscillations specifically impact spatial attention neuronal processes, and thereby behavioral performance.

a

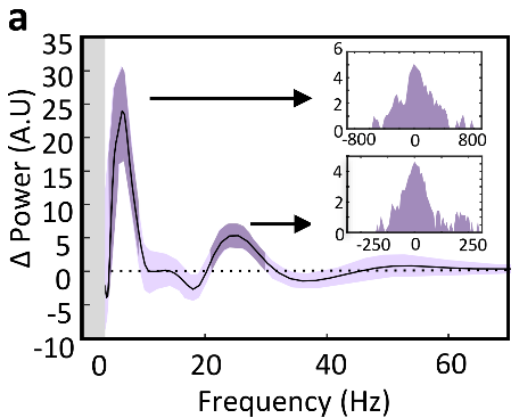

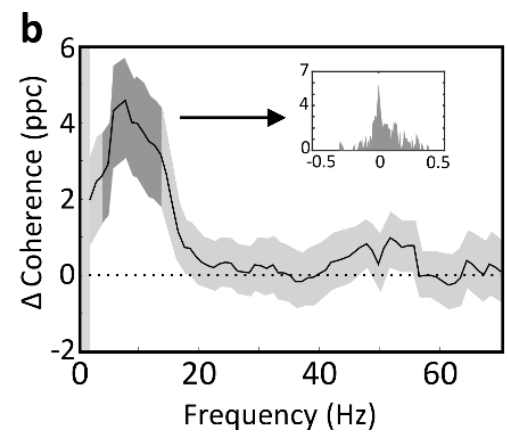

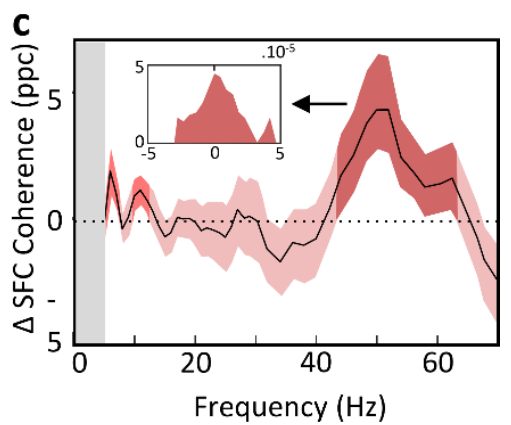

Figure 5: Ultra slow oscillations in MUA and LFP attention-related content coincides with significant changes in LFP power (A), LFP coherency (B) and spike field coherency (C). All metrics are extracted over the $1000 \mathrm{~ms}$ preceding target presentation. For each metric, the main plot represents the difference between the metric obtained for trials at the peak of the slow oscillations vs. trials at the trough of the slow oscillations, averaged over all 
recording signals ( $n=912$ channels, mean + - s.e., above 95 C.I in darker shades). Insets represent the distribution of the modulation index of each metric between the peak and trough of the slow oscillation trials, averaged over significance epoch (black arrow), over all recorded signals ( $n=912$ channels).

324 In addition, we observe during the same trial epoch (1000ms before target presentation), enhanced 325 coherency across LFPs, in the correct trials located at the peak relative to the trough of the ultra-slow oscillations, as assessed from a coherency measure (pairwise phase consistency). This enhanced coherency is specifically observed in the alpha range (average peak $=9.25 \mathrm{~Hz}+-7.35$ s.e., Fig. $5 b$, significance assessed against 95\% C.I.). The majority of LFP pairs are up-modulated in the peak relative to the trough of the oscillation cycles (Figure 5b, inset). This suggests that the FEF receives long-range signals, modulating overall alpha coherency of the LFPs all across the FEF map, bilaterally.

We further show that spike field-coherency estimated during the same trial epochs as LFP power and coherency, are enhanced on the correct trials located at the peak relative to the trough of the ultraslow oscillations in three specific frequency ranges (Fig. 5c, significance assessed against 95\% C.I.): the theta (average peak=6.25 Hz+- 0.75 s.e.), alpha (average peak=11 Hz +- 1.30 s.e.), and gamma range

335 (average peak=50.75 Hz +- 6.75 s.e.). Changes in spike-field coherence in the gamma band are reliably 336 associated with changes in attentional processes orientation(Chalk et al., 2010; Fiebelkorn et al., 2019;

337 Fries, 2015a; Fries et al., 2001).

338 Overall, these results indicate that the observed ultra-slow fluctuations in MUA-related attentional 339 processes are subsequent to long-range changes impacting prefrontal LFPs, and are locally mediated 340 through specific changes in spike-field coherence mechanisms.

\section{Human behavioral performance and pupil size fluctuates at 4 to 7 cycles per hour}

342 In order to test whether the ultra-slow rhythm described above is specific to high attentional demand 343 tasks, we had 30 human subjects detect a shivering Gabor in the midst of slowly drifting Gabors of 344 variable orientations for 75 minutes with no interruption. Figure 6a represents the running estimate of 345 an exemplar subject detection times (i.e. time taken by the subject to identify a new shivering Gabor)

346 across a single session. As seen in the behavioral performance of monkeys, the detection times fluctuate 347 by up to 15 seconds. A wavelet analysis reveals a significant oscillatory peak at 5.85 cycles per hour 348 (Fig. 6b, permutation test, $\mathrm{p}<0.05$ ). This specific rhythm in subjects' detection time was consistently 349 identified across subjects, at an average frequency of $5.96+-0.25$ cycles per hour (Fig. 6e, orange, mean $350+$ - s.e., $n=35$ ). 
351 Changes in pupil size is associated with a broad range of cognitive processes(Beatty and Lucero-

352 Wagoner, 2000; Sirois and Brisson, 2014; van der Wel and van Steenbergen, 2018), including attention(B

353 et al., 2012), and working memory(Just et al., 2003; Kramer, 1990). In addition, increase in pupil size has

354 been taken as an indirect index of the noradrenergic function(Dragone et al., 2018; Reynaud et al.,

355 2019). Accordingly, in a subset of subjects ( $n=19)$, we recorded pupil size while performing the task.

356 Figure $6 \mathrm{~b}$ represents the running estimate of an exemplar subject pupil size across a recording session

357 (same exemplar session as Fig. 6a). A wavelet analysis reveals a significant frequency peak at 5.7 cycles

358 per hour (Fig. 6d, permutation test, p<0.05), consistently identified across subjects, at an average

359 frequency of $5.56+-0.43$ cycles per hour (Fig. 6e, pink, mean +- s.e., n=19). Detection times and pupil

360 sizes peak frequency distributions across subjects were highly similar (Ranksum test for equal mean,

$361 p>0.57, n=19)$.

a

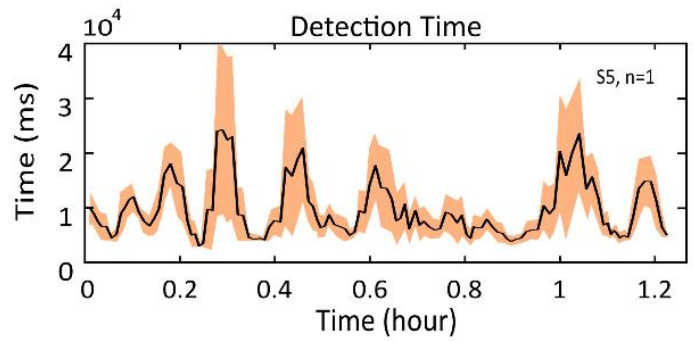

C
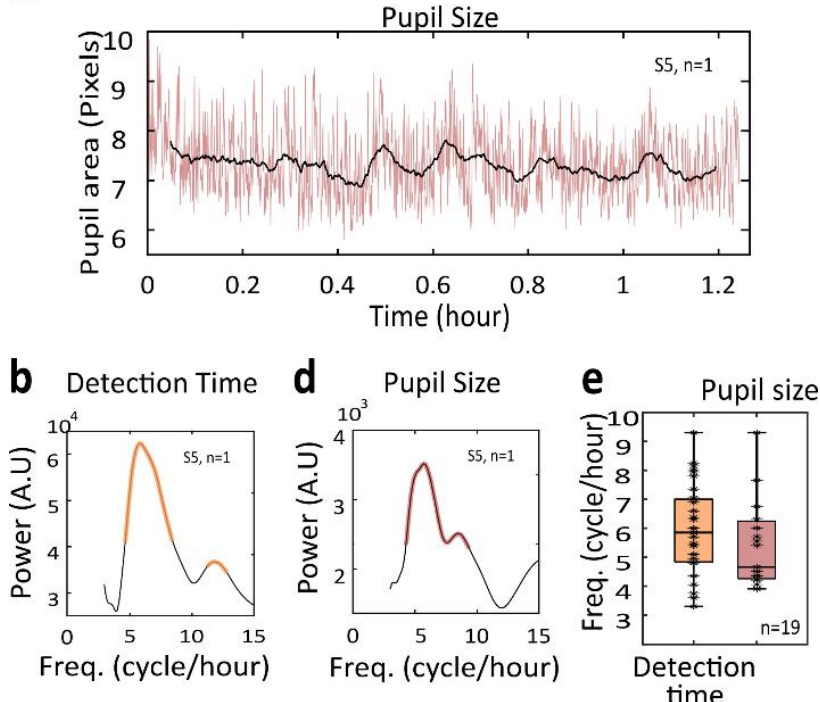

Figure 6: Ultra-slow rhythmic fluctuations in behavioral performance and pupil size in human subjects during sustained cognitive activity. (a) Detection time estimates throughout session time (mean +/-s.e.) for the exemplar subject S5 and (b) corresponding power spectrum (orange, test for random permutations, $p<0.05$ ). (c) Pupil area estimates throughout session time (black line: convoluted pupil size, pink line: raw pupil size data) for the exemplar subject S5 and (d) corresponding power spectrum (pink, test for random permutations, $\mathrm{p}<0.05$ ). (e) Distribution of ultra-slow oscillatory peaks across all 
subjects (median +/- s.e.), for detection times (orange) and corresponding pupil size (pink), all frequency peaks significant in all subjects (random permutation $\mathrm{p}<0.05$ ).

\section{Discussion}

372 Overall, we show that when performing an attentional task for one hour or more, the behavior of both

373 humans and non-human primates consistently fluctuates between periods of high and low performance

374 at a rhythm of 4 to 7 cycles per hour (every 9 to 15 minutes). These behavioral fluctuations are observed

375 in both a difficult (in non-human primates) and an easy (in humans) attentional task, indicating that

376 these fluctuations are not dependent on the task demand. At the physiological level, these epochs of

377 high and low performance coincide with fluctuations in pupil diameter, indicating underlying changes

378 in arousal and information-processing load. Accordingly, we show that these behavioral fluctuations

379 coincide, at the neurophysiological level, with fluctuations in the attention and perception coding

380 accuracy of prefrontal neuronal populations. We further identify specific markers of these fluctuations

381 in LFP power, LFP coherence and spike-field coherence, suggesting long-range rhythmic modulatory

382 inputs to the prefrontal cortex rather than a local prefrontal origin.

\section{Novel ultra-slow rhythmic fluctuations in behavior and neuronal processes}

384 Ongoing fluctuations in neural activity that are independent of sensory processes have been described 385 in the brain at multiple time scales(Arieli et al., 1996; Aston-Jones and Cohen, 2005; Beaman et al., 2017; 386 Cowley et al., 2020; Engel et al., 2016; Gaillard et al., 2020b; McGinley et al., 2015, 2015, 2015; Milton et al., 2020; Oby et al., 2019; Okun et al., 2015, 2019; Reimer et al., 2014; Stringer et al., 2019; Vinck et al., 2015; Wasmuht et al., 2019). These fluctuations are shown to correlate with behavioral and physiological variables such as pupil diameter(Ebitz and Moore, 2017; Gutnisky et al., 2017; Joshi et al., 2016; McGinley et al., 2015; Nassar et al., 2012; Okun et al., 2015; Stringer et al., 2019; Vinck et al., 391 2015), eye movements(Leopold and Logothetis, 1998; Steinmetz and Moore, 2014), 392 wakefulness(McGinley et al., 2015; Milton et al., 2020; Reimer et al., 2014), or attention(Engel et al., 393 2016; Huang and Elhilali; Moore and Zirnsak, 2017; Rabinowitz et al., 2015; Snyder et al., 2016).

394 Some of these fluctuations in behavior and neuronal processes are rhythmic, spanning a wide range of 395 frequencies from $0.01 \mathrm{~Hz}$ (Chan et al., 2017; Klein and Armitage, 1979; Palva and Palva, 2011), up to $396100 \mathrm{~Hz}$ (100 cycles per second(Fries, 2005; Jia and Kohn, 2011)). Attentional and perceptual cortical 397 processes have specifically been associated with theta frequencies (3-6 Hz(Dugué et al., 2014, 2016; 398 Fiebelkorn et al., 2018a) as well as alpha (7-12 Hz(Gaillard et al., 2020b)), beta (18-25 Hz(Hassen et al., 399 2019; Vernet et al., 2019)) and gamma frequencies (25-100 Hz(Fries, 2015a)). For example, in the 400 primate primary visual cortex, Engel et al. (2016) describe an alternation of on and off spiking episodes 401 of $149 \mathrm{~ms}$ and $100 \mathrm{~ms}$ average duration respectively(Engel et al., 2016). This pattern of alternation is 
402 similar to that described during sleep or anesthesia. More recently, Gaillard et al., (2020) describe 403 rhythmic fluctuations in prefrontal attention-related neuronal activity in the 7-12 Hz range(Gaillard et 404 al., 2020b). McGinley et al (2015) show that cortical membrane potential in the mice auditory cortex 405 co-varies with pupil size at two different signature frequencies of $0.03 \mathrm{~Hz}$ and $0.3 \mathrm{~Hz}$ respectively, as a 406 function of general arousal(McGinley et al., 2015). All of these variations are predictive of optimal neuronal and behavioral processes, but all happen at a much faster time scale than the one described in the present study. A recent study describes widespread changes in neuronal dynamics in the dIPFC of the freely moving macaque monkey(Milton et al., 2020). This study identifies transitions between desynchronized neuronal states during active wakefulness and synchronized neuronal states during quiet wakefulness. In contrast with our observations, synchronous activity was associated with a significant decrease in spiking rates and an increase in the power of the slow LFP oscillations in the 0.5$10 \mathrm{~Hz}$ range. These transitions were highly correlated with spontaneous motion and took place every 30 minutes to 60 minutes, thus at a slower rate than described here. At closer inspection of the Milton et al. data(Milton et al., 2020), however, faster transitions can be seen, predominantly in the wake state, at the same rhythm as described here. This suggests that the rhythmic fluctuations we describe interact with wake/rest transitions but stem from an independent neuronal system mechanism. This will have

418 to be further explored experimentally.

419 Up to now, the description of slow rhythms in the range of the hour has been missing. This is due to the 420 fact that data is often collected in short blocks of 5 to 20 minutes and subjects are allowed to rest in 421 between, thus disrupting the temporal structure in sustained workload processes at each pause. By 422 recording behavioral performance during sessions lasting from 1 to 4 hours (with no externally 423 generated interruptions), we report rhythmic fluctuations in behavioral performance in the range of 4 424 to 7 cycles per hour. These fluctuations are consistent across individuals and species. In addition, they are independent from task attentional demand (as they can be reported in both a low and a high attentional demand task) and subjects' expertise (as they can be reported both in naïve human subjects and over-trained macaques). Importantly, they occur in the absence of any kind of monotonic change in behavioral performance as time goes by, in contrast with what has been described by others(Cowley et al., 2020). This thus goes against current theories hypothesizing a decrement of performance under sustained-attention constraints (e.g. the resource-control hypothesis(Thomson et al., 2015)). Over

431 longer periods of days and even weeks, mental effort and cognitive fatigue have been shown to 432 progressively degrade cognitive performance(Lockley et al., 2004; Proctor et al., 1996; Virtanen et al., 433 2009). It will be crucial to understand how this decay in cognitive performance interacts with the 434 behavioral ultra-slow rhythms we describe here and whether there is a functional link between these 435 two observations. 


\section{Neuronal correlates of the ultra-slow fluctuations in behavioral performance}

437 We specifically show that both prefrontal attentional and perceptual neuronal processes are altered during the ultra-slow rhythmic fluctuations, directly impacting behavioral performance. The net effect of the described rhythmic fluctuations is a higher attentional and perceptual information content, i.e. neuronal processes closer to optimality, during high behavioral performance epochs compared to low performance epochs. Importantly, these changes take place in the absence of significant overall changes in neuronal spiking rate. They thus are not associated with a global variation in neuronal population excitability(Milton et al., 2020), but rather with a change in overall population informational coding accuracy.

We find that these rhythmic modulations of behavior also impact several neuronal markers that have been associated with attentional and perceptual processes. First, we report an enhancement of the power of prefrontal LFP activity in the alpha(Bollimunta et al., 2008; Gaillard et al., 2020a; Mo et al., 2011) (6-9Hz) and beta(Buschman and Miller, 2009; Fiebelkorn et al., 2018b; Siegel et al., 2008)

449 frequencies $(20-30 \mathrm{~Hz})$. Second, we show that on high behavioral performance epochs, LFP alpha 450 coherency is higher than on low behavioral performance epochs. Signal coherency within and between 451 brain areas is proposed as a key mechanism at the core of brain computing functions and supports 452 information/stimulus processing(Fries, 2015b), accounting for network connectivity(Vinck et al., 2010), 453 and changes in behavioral performance(Zareian et al., 2018, 2020). Last, we report changes in spike 454 field coherency within the prefrontal cortex between the epochs of high vs. low behavioral performance, in the same frequency ranges as reported by others during attention orientation(Fries et al., 2008; Gregoriou et al., 2009b; Paneri and Gregoriou, 2017), thus pointing to a direct modulation of attentional mechanisms.

Overall, this supports the idea that epochs of high vs low behavioral performance coincide with specific changes in the attention and perception processes. These changes arise from long-range mechanisms supported by LFP coherency and LFP power content in specific frequency bands repeatedly associated with attentional processes, as well as from local changes in spike-field coherence in these very same frequency bands. The origin of these fluctuations, whether arising from global brain network configuration, or from a specific brain region controller, remains to be explored. It is unclear whether the observed very slow rhythmic processes only impact the functions being recruited by the task, namely attention and perception (and hence specifically the functional brain network subserving this

466 function), or whether theses fluctuations impact all cognitive performances, irrespective of the task.

467 Again, this will have to be further explored. 
During the execution of cognitive tasks in humans, "task-off" or "mind-wondering" states (in contraposition to "task-on" periods) can be observed(Compton et al., 2019; Smallwood and Schooler,

471 2015). These "task-off" epochs have been associated with specific neural signatures such as increased 472 scalp alpha activity(van Dijk et al., 2008) and an enhanced activation of the default-mode473 network(Knyazev et al., 2011; Mo et al., 2013). These states of attentional lapses have been explained 474 in the context of resource-control theory(Thomson et al., 2015), that posits that (i) an executive control 475 is required to sustain active goal maintenance, (ii) the amount of attentional resources available for any 476 given individual is fixed and (iii) attentional resources are consumed both during task-on and task-off 477 states. As a consequence, this model predicts that executive control wanes over task-on periods, 478 resulting in performance-costs in task-off periods.

479 Our data show oscillations in behavioral performance. These behavioral oscillations coincide with 480 fluctuations in prefrontal attentional coding accuracy (or resources). As a result, they can be seen as 481 alternations between task-on and task-off periods. However, attentional resources alternate between 482 states of depletion (transitory waning) and states of efficient processing (restoration), instead of waning 483 with time, as initially predicted by the resource control theory. These fluctuations in behavior also 484 coincide with fluctuations in pupil size. This physiological parameter has been shown to depend on the 485 noradrenergic system(Dragone et al., 2018; Reynaud et al., 2019) and to be altered in patients with 486 attention disabilities(Beane and Marrocco, 2004). The rhythmic transitions between task-on and task487 off epochs and associated attentional and perceptual processes could thus be under the direct influence 488 of the Locus Coeruleus and noradrenergic neuromodulation. This will have to be further explored.

\section{Possible origin of ultra-slow rhythmic fluctuations}

490 Changes in brain states have been reported in the wake state and during the different sleep cycles or 491 during states of altered consciousness such as anesthesia(Barttfeld et al., 2015; Demertzi et al., 492 2019). None of these changes take place at frequencies nor coincide with as specific neuronal correlates 493 in LFP power, LFP coherency and spike field coupling as described in the present work. Thus, the 494 mechanistic origin of the behavioral and neurophysiological rhythmic fluctuations reported in this 495 manuscript remains elusive. One possible explanation is that these fluctuations arise as a consequence 496 of cognitive fatigue, in which one can expect a partial impairment of the neuronal processes supporting 497 the attentional function, thus limiting behavioral performance by decreasing alertness and information 498 processing. This hypothesis would predict alternations of metabolic depletion and regeneration, and a specific impact on the recruited functional network rather than on the entire brain. A second explanation for this alternation between optimal and suboptimal cognitive abilities could be an 
accomplish an optimal global timing. If they run too fast too often, they risk to collapse out of exhaustion. If they run too slow, they will not maximize their reward, be it a prize or an individual record. In a similar way, ultra-slow oscillations can allow to maintain an acceptable level of cognitive performance across very long periods of time without depleting the entire brain cognitive resources, which would eventually result in a complete shutdown of all processes. A third hypothesis is that multilevel oscillatory activity could arise from the specific organization of the primate brain. Specifically, many cognitive functions often rely on complex neuronal networks involving multiple areas related to each other by complex feedforward and feedback connections. Indeed, reports show that synchronized brain rhythms could arise in densely wired systems (such as the primate brain) in a small-world way(Kim and Lim, 2013; Li et al., 2015) and propose that a scale-free network structure of the brain could be responsible for large scale rhythmic activity (Mi et al., 2013). Such multi-scaled and hubs organized structures are described to be highly adapted against local impairment or lesion. Modelling work would help support or infirm this possibility. A fourth hypothesis would be a neuromodulatory origin. The locus coeruleus, which distributes norepinephrine all across the brain through widespread projections(AstonJones and Cohen, 2005) modulates arousal. In particular, its neuronal activity has been linked to variability in pupil size(Joshi et al., 2016; Liu et al., 2017; McGinley et al., 2015) and behavioral performance(Aston-Jones and Cohen, 2005; Eldar et al., 2013). Another candidate is acetylcholine, which is released by the basal forebrain, and impacts pupil size as well as locomotion(Everitt and Robbins, 1997; McCormick, 1992; McGinley et al., 2015; Yüzgeç et al., 2018). These multiple hypotheses will have to be evaluated experimentally.

\section{Ultra-slow rhythms and attentional reallocation}

524 Tradeoff between exploratory and exploitation is crucial for optimal behavior. For example, a bee can 525 keep collecting pollen from the same flower (exploitation), which comes at a cost of lower harvest (all pollen will end up being collected), or move (exploration) from one flower to the next(Kembro et al.,

527 2019) (exploration), which comes at a strong energetic cost (moving from one flower to the next).

528 Allocation of human brain attentional mechanisms is proposed to alternate between exploration and 529 exploitation strategies(Gaillard et al., 2020a; VanRullen, 2018). The rhythmic drop in the accuracy of 530 ongoing cognitive processes that we report here might actually leave an open window for the subject 531 to shift cognitive resources from the ongoing process to another process. From an ecological 532 perspective, this would force on the subject a kind of cognitive flexibility, possibly originating from 533 general large-scale transitions in brain functional networks. Such transitions have been described at 534 lower time scales during normal brain states and states of altered consciousness(Huang et al., 2020).

\section{Conclusion}


536 Modern societies and the rise of assistive technologies result in a whole new framework where human

537 cognitive production is shifted from low cognitive demand tasks to sustained demanding cognitive tasks

538 over long time periods. It is thus crucial to understand how the human brain is able to produce and

539 sustain high demand cognitive processes efficiently, without producing and accumulating errors. In this

540 context, the slow rhythmic behavioral and cognitive fluctuations described here are expected to instruct

541 on how to optimize work and school environments, in a way that is more adapted to brain physiology.

542 In addition, they are a promising targets for pharmacological or neuro-modulatory enhancement using

543 behavioral training, transcranial magnetic stimulation or neurofeedback(Bagherzadeh et al., 2020;

544 Dugué and VanRullen, 2017; Dugué et al., 2014; Horschig et al., 2015; Ros et al., 2017; Saj et al., 2018).

545 These methods have up to now targeted infra-second rhythms. Applying them to the described ultra-

546 slow behavioral and cognitive rhythm is expected to open interesting perspectives in the field of human

547 productivity, learning and teaching. 
bioRxiv preprint doi: https://doi.org/10.1101/2021.05.26.445730; this version posted May 26, 2021. The copyright holder for this preprint (which was not certified by peer review) is the author/funder. All rights reserved. No reuse allowed without permission.

\section{Acknowledgments}

550 S.B.H was supported by ERC Brain3.0 \#681978, ANR-11-BSV4-0011 \& ANR-14-ASTR-0011-01, LABEX CORTEX

551 funding (ANR-11-LABX-0042) from the Université de Lyon, within the program Investissements d'Avenir (ANR-11-

552 IDEX-0007) operated by the French National Research Agency (ANR). C.D.S., C.G., J.A. and C.L. were supported by

553 ERC Brain3.0 \#681978. We thank research engineer Serge Pinède for technical support and Jean-Luc Charieau and

554 Fidji Francioly for animal care. All procedures were approved by the local animal care committee (C2EA42-13-02-

555 0401-01) and the Ministry of research, in compliance with the European Community Council, Directive

556 2010/63/UE on Animal Care. All human experiments were performed in accordance with relevant guidelines and

557 regulations (the declaration of Helsinski). All experimental protocols were approved by the CNRS (research

558 institution acting as promotor) and the CPP-Sud-Est (acting as licensing committee). The project authorization is

$559 \quad$ \#ID RCB 2017-A03612-51.

\section{Author contributions}

561 Conceptualization, C.G., C.D.S. and S.B.H.; Data Acquisition, S.B.H.H., F.D.B., J.A., C.L., C.Z.; Methodology, C.G., 562 C.D.S and S.B.H.; Investigation, C.G., C.D.S. and S.B.H.; Writing - Original Draft, C.G., C.D.S and S.B.H.; Writing 563 Review \& Editing, C.G., C.D.S., J.A. and S.B.H.; Funding Acquisition, S.B.H.; Supervision, S.B.H. 
567 All procedures were in compliance with the guidelines of European Community on animal care 568 (Directive 2010/63/UE of the European Parliament and the Council of 22 September 2010 on the 569 protection of animals used for scientific purposes) and authorized by the French Committee on the 570 Ethics of Experiments in Animals (C2EA) CELYNE registered at the national level as C2EA number 42 571 (protocole C2EA42-13-02-0401-01).

\section{Monkey experiments - Subjects and surgical procedure}

573 Two male rhesus monkeys (Macaca mulatta) weighing between 6-8 $\mathrm{kg}$ underwent a surgery during 574 which they were implanted with two MRI compatible PEEK recording chambers placed over the left and 575 the right FEF hemispheres respectively (figure 1A), as well as a head fixation post. Gas anesthesia was 576 carried out using Vet-Flurane, 0.5 - 2\% (Isofluranum 100\%) following an induction with Zolétil 100 577 (Tiletamine at $50 \mathrm{mg} / \mathrm{ml}, 15 \mathrm{mg} / \mathrm{kg}$ and Zolazepam, at $50 \mathrm{mg} / \mathrm{ml}, 15 \mathrm{mg} / \mathrm{kg}$ ). Post-surgery pain was 578 controlled with a morphine pain-killer (Buprecare, buprenorphine at $0.3 \mathrm{mg} / \mathrm{ml}, 0.01 \mathrm{mg} / \mathrm{kg}$ ), 3 injections 579 at 6 hours interval (first injection at the beginning of the surgery) and a full antibiotic coverage was 580 provided with Baytril 5\% (a long action large spectrum antibiotic, Enrofloxacin $0.5 \mathrm{mg} / \mathrm{ml}$ ) at 2.5mg/ $\mathrm{kg}$, 581 one injection during the surgery and thereafter one each day during 10 days. A $0.6 \mathrm{~mm}$ isomorphic 582 anatomical MRI scan was acquired post surgically on a 1.5T Siemens Sonata MRI scanner, while a high583 contrast oil filled grid (mesh of holes at a resolution of $1 \mathrm{~mm} \times 1 \mathrm{~mm}$ ) was placed in each recording 584 chamber, in the same orientation as the final recording grid. This allowed a precise localization of the 585 arcuate sulcus and surrounding gray matter underneath each of the recording chambers. The FEF was 586 defined as the anterior bank of the arcuate sulcus and we specifically targeted those sites in which a

587 significant visual and/or oculomotor activity was observed during a memory guided saccade task at 10 588 to $15^{\circ}$ of eccentricity from the fixation point (figure $1 \mathrm{~A}$ ). In order to maximize task-related neuronal 589 information at each of the 24-contacts of the recording probes, we only recorded from sites with task590 related activity observed continuously over at least $3 \mathrm{~mm}$ of depth.

\section{Monkey experiments - Behavioral task and Experimental setup}

592 The task is a 100\% validity endogenous cued target detection task (fig 1a). The animals were placed in 593 front of a PC monitor (1920×1200 pixels and a refresh rate of $60 \mathrm{HZ})$, at a distance of $57 \mathrm{~cm}$, with their 594 heads fixed. The stimuli presentation and behavioral responses were controlled using Presentation 595 (Neurobehavioral systems ${ }^{\circledR}$, https://www.neurobs.com/). To start a trial, the bar placed in front of the 596 animal's chair had to be held by the monkeys, thus interrupting an infrared beam. The onset of a central 597 blue fixation cross $\left(\right.$ size $\left.0.7^{\circ} \times 0.7^{\circ}\right)$ instructed the monkeys to maintain eye position inside a $2^{\circ} \times 2^{\circ}$ 
window, defined around the fixation cross. To avoid the abort of the ongoing trial, fixation had to be

599 maintained throughout trial duration. Eye fixation was controlled thanks to a video eye tracker (Iscan ${ }^{\mathrm{TM}}$ ).

600 Four gray square landmarks $\left(\mathrm{LMs}-\right.$ size $\left.0.5^{\circ} \times 0.5^{\circ}\right)$ were displayed, all throughout the trial, at the four

601 corners of a $20^{\circ} \times 20^{\circ}$ hypothetical square centered onto the fixation cross. Thus, the four LMs (up-right,

602 up-left, down-left, down-right) were placed at the same distance from the center of the screen having

603 an eccentricity of $14^{\circ}$ (absolute $x$ - and $y$-deviation from the center of the screen of $10^{\circ}$ ). After a variable

604 delay from fixation onset, ranging between $700-1200 \mathrm{~ms}$, a small green square (cue - size $0.2^{\circ} \times 0.2^{\circ}$ )

605 was presented, for $350 \mathrm{~ms}$, close to the fixation cross (at $0.3^{\circ}$ ) in the direction of one of the LMs.

606 Monkeys were rewarded for detecting a subtle change in luminosity of this cued LM. The change in

607 target luminosity occurred unpredictably between $350-3300 \mathrm{~ms}$ from the cue off time. In order to

608 receive a reward (drop of juice), the monkeys were required to release the bar in a limited time window

609 (150- $750 \mathrm{~ms})$ after the target onset (Hit trial). In order to make sure that the monkeys did use the cue

610 instruction, on half of the trials, distractors were presented during the cue to target interval. Two types

611 of distractors could be presented: (i) uncued landmark distractor trials (33\% of trials with distractor);

612 these corresponded to a change in luminosity, identical to the awaited target luminosity change, and

613 could take place equiprobably at any of the uncued LMs; (ii) workspace distractor trials (67\% of trials

614 with distractor); these corresponded to a small square presented randomly in the workspace defined

615 by the four landmarks. The contrast of the square with respect to the background was the same as the

616 contrast of the target against the LM; when presented at the same radial eccentricity as the LMs, the

617 workspace distractor had the same size as the landmarks; for smaller eccentricities, the size of the

618 workspace distractor was adjusted for cortical magnification such that it activated an equivalent cortical

619 surface at all eccentricities. The monkeys had to ignore all of these distractors. Responding to any of

620 them interrupted the trial. If the response occurred in the same response window as for correct

621 detection trials (150 - $750 \mathrm{~ms}$ ), the trial was counted as a false alarm (FA) trial. Failing to respond to the

622 target (Miss) similarly aborted the ongoing trial. Overall, data was collected for 19 sessions (M1 10

623 Sessions, M2 9 Sessions). The behavioral performance of each animal on correct trials is presented in

624 figure $1 b$, depending on whether distractors were present or not.

\section{Monkey experiments - Electrophysiological recording}

626 Bilateral simultaneous recordings in the two frontal eye fields (FEF) were carried out using two 24

627 contacts Plexon U-probes (fig. 1b). The contacts had an interspacing distance of $250 \mu \mathrm{m}$. Neural data

628 was acquired with the Plexon Omniplex ${ }^{\circledR}$ neuronal data acquisition system. The data was amplified 400

629 times and digitized at 40,000 $\mathrm{Hz}$. A threshold defining the multi-unit activity (MUA) was applied

630 independently for each recording contact and each session before the actual task-related recordings

631 started. 


\section{Monkey experiments - Behavioral performance as a function of session time}

633 Hit and Miss trials were compiled relative to their absolute time position in the session. Behavioral 634 performance, defined as hits/(hits+misses) was computed on successive sets of 50 trials, corresponding 635 to an average time period of $5.83 \pm 1.1 \mathrm{~min}$. This procedure was iterated over the entire length of the 636 session in 1 trial steps. Time sampling variability (due to variability in trial length and reaction times) was 637 compensated by assigning, to each running behavioral performance estimate, its corresponding 638 Gaussian mean trial time relative to the session ( $\sigma=$ length of testing set/ 6 , here $\sigma=4.166$ ). This procedure resulted in a time series representing running behavioral performance as a function of time in the session.

Monkey experiments - Signal frequency analyses of behavioral performance time series

642

643

644

645

646

647

648

649

650

651

652

653

654

655

656

657

658

659

660

661

662

663

664

Behavioral performance time series were interpolated (cubic spline function) for each independent session. Across sessions, this resulted in a sampling frequency of $1538.4 \pm 232$ samples/hour for an average session duration of 3.526 hours \pm 0.6796 . Specifically, median task duration was 3.94 hours for monkey M1 (s.e. = 0.66) and 2.99 hours for monkey M2 (s.e. = 0.20). These parameters allow the description of frequencies ranging from $0.29 \pm 0.515$ to $769 \pm 116$ cycles per hour. The spectral analysis of this time series was performed on detrended data using a Morlet Wavelet transform. Standard error represented on the power spectrum is calculated along the time dimension of the wavelet analysis. We established statistical significance by reshuffling trials presentation time across the session (1000 times) and generating a random distribution of signal spectral content. Significance was then computed by comparing each session power spectrum to its corresponding 95\% confidence interval.

\section{Monkey experiments - Behavioral outcome decoding procedure}

MUA and LFP recorded during the task were aligned on target presentation time and sorted according to monkey's behavioral response (Correct trials or misses). A regularized linear decoder was used to associate, the neuronal activity estimated on a $200 \mathrm{~ms}$ post-target interval (corresponding to the FEF population responses to the visual target presentation) to the two possible behavioral response of subject. During training, the input to the classifier was a 48 elements by $\mathrm{N}$-trial matrix corresponding to the average neuronal response on each recording channel for the time interval of interest for each of the $\mathrm{N}$ training trials. The imposed output of the classifier was the behavioral response of these $\mathrm{N}$ training trials. During testing, the output of the classifier was estimated for a 48 element vector corresponding to the average neuronal response on each recording channel for the time interval of interest on a testing trial, new to the classifier. This output can be read as a class output, corresponding to one of the two behavioral responses. Supplementary Fig. 1 presents for MUA and LFP signal based decoder accuracies to classify behavioral outcome (hit or miss). Data were averaged on 19 sessions and compared to 1000 
random distribution based on trials labels shuffling for each session. To quantify prefrontal encoding stability across session time, we performed cross-temporal decoding analyses along the entire length of the session. A classifier was trained on a given training set of 200 trials from a specific time in the session. For each individual training set, behavioral response categories (correct or miss) were randomly equalized across trials (100 repetitions). Testing was then performed on the next 50 trials and classification performance was computed on these 50 trials. This procedure was iterated by steps of 1 correct trial at a time, to cover the entire session trial range. The classification performance of each testing set of 50 trials was assigned to the Gaussian mean of the presentation time of these 50 trials relative to the session ( $\sigma=$ length of testing set/ 6 , here $\sigma=8.32$ ). This procedure resulted in a time series representing running classification performance as a function of session time.

\section{Monkey experiments - Attention $(x, y)$ position decoding procedure}

676 Similarly, to the decoding procedure described in the previous section, a regularized linear decoder was 677 used to associate, on correct trials, the neuronal activity estimated on a 400ms pre-target interval to 678 the expected position of subject's attention(Astrand et al., 2016; De Sousa et al., 2021; Gaillard et al., 679 2020a). During training, the input to the classifier was a 48 elements by $\mathrm{N}$-trial matrix corresponding to 680 the average neuronal response on each recording channel for the time interval of interest for each of 681 the $N$ training trials. The imposed output of the classifier was the $(x, y)$ coordinates of the cued landmark 682 for each of these $N$ training trials. During testing, the output of the classifier was estimated for a 48 683 element vector corresponding to the average neuronal response on each recording channel for the time 684 interval of interest on a testing trial, new to the classifier. This output can be read as a continuous $(x, y)$ 685 estimate of attention location or as a class output, corresponding to one of the four possible visual 686 quadrants (Astrand et al., 2016; Gaillard et al., 2020a).

\section{Monkey experiments - Attention classification procedure}

688 To quantify prefrontal attention stability across session time, we also performed cross-temporal 689 decoding analyses along the length of the session. A classifier was trained on a given training set of 200 690 successive correct trials from a specific time in the session. For each individual training set, cued spatial 691 categories (position) were randomly equalized across trials (100 repetitions). Testing was then performed on the next 25 correct trials and classification performance was computed on these 25 trials.

693 This procedure was iterated by steps of 1 correct trial at a time, to cover the entire session trial range. 694 Because of the randomized structure of the task and the variable rate of incorrect trials over session 695 time, the actual duration of each training set varied within and across session (4.550 $\pm 0.547 \mathrm{~min})$. To minimize this temporal sampling variability, and have a robust estimate of mean trial distribution, the 
presentation time of these 25 trials relative to the session ( $\sigma=$ length of testing set $/ 6$, here $\sigma=4.166$ ). This procedure resulted in a time series representing running classification performance as a function of session time.

\section{1}

\section{Monkey experiments - Signal frequency analyses of classification performance time series}

702 Each independent session classification performance time series was interpolated (cubic spline 703 function). This resulted in a fixed sampling frequency of $1000 \pm 179$ sample/hour for an average session 704 duration of 3.526 hours \pm 0.6796 . These parameters allow the description of frequencies ranging from $7050.29 \pm 0.515$ to $500 \pm 89.97$ cycle per hour. The spectral analysis of this time series was performed on 706 detrended data using a Morlet Wavelet transform.

\section{Monkey experiments - Phase analysis}

708 For each session, phase difference distributions was obtained using a cross wavelet transform analysis 709 (Grinsted et al., 2004). Specifically, cross wavelet transform computes at each frequency and each 710 timestamp the relative phase difference between both time series at the same frequency band. Phases 711 distribution were compared across all sessions and both subjects using non parametric circular Kuiper's 712 test for equal distributions. Phase comparison were performed between time series constructed from 713 comparable timestamps only, defined as follows. Specifically, behavioral performance and decoded 714 behavioral neuronal time series are both computed over hit and miss trials. They are thus estimated at 715 exactly the same timestamps in the session (condition 1). In contrast, decoded attentional time series 716 for MUA or LFP are assessed on correct trials. These time series are thus estimated at exactly the same 717 timestamps (condition 2). However, the timestamps estimated in condition 1 and condition 2 are 718 different. Indeed, the presence of miss trials of varying frequency of occurrence leads to local time 719 compression or expansion of one timestamp series relative to the other. Because phase estimation is a 720 very sensitive metric, phase analysis could only be performed across time series estimated from the 721 same timestamps.

\section{Monkey experiments - Performance at optimal phase computation}

723 Average performance at optimal phase was estimated independently computed for each session then 724 averaged. Specifically, for each session, identified oscillatory cycle was independently subdivided into 72517 phase bins, and a phase to behavioral performance function was calculated. Optimal phase was 726 defined for each session as the absolute phase of the cycle maximizing subject performances(Busch and 727 VanRullen, 2010; Dugué et al., 2014; Gaillard et al., 2020a). Subsequently, phase to behavioral phases 728 functions were aligned across sessions and averaged to compute averaged relative increase in 729 performance at optimal versus suboptimal phase of the oscillation. Central points ( 0 phase, by definition maximal) were removed from statistical all analysis. 


\section{Monkey experiments - LFP power and coherency analysis}

732 LFP power spectra were estimated for each channel and each trial using Morlet Wavelet transform over 733 an attentional period ranging from $500 \mathrm{~ms}$ post cue presentation to $2000 \mathrm{~ms}$ (1.5s epoch). Coherency 734 was computed separately for each hemisphere to avoid possible inter-hemispheric phase differences in 735 LFP frequency content. Coherency was computed using pairwise phase consistency (PPC) performed on 736 a 1 second time epoch (500 ms post cue presentation to $1500 \mathrm{~ms}$ ) and was based on the fieldtrip 737 ft_connectivityanalysis function(Oostenveld et al., 2011).

\section{Monkey experiments - Spike LFP phase coupling}

739 For each spike within a 1s time window prior to target presentation (on trials with cue to target intervals 740 of $1500 \mathrm{~ms}$ or higher), we computed the LFP phase estimates at spike time, using pairwise phase consistency estimation (PPC). PPC allows to compute spike LFP phase coupling (SFC) independently of spike and trial number. This was performed using the fieldtrip ft_spiketriggeredspectrummatlab function. In order to adapt SFC computation to frequency bands of interest, we used 4, 6 and 8 cycles

744 per frequency to compute frequency phases estimates in respectively the $2-20 \mathrm{~Hz}, 20-50 \mathrm{~Hz}$ and $50-70 \mathrm{~Hz}$

745 frequency bands.

\section{Monkey experiments - Peak and trough session oscillation estimation}

747 For each session, peak and trough trials were identified based on time series rhythmic variations in 748 session time. First, the behavioral signal was filtered at the specific low-frequency dominating the 749 behavioral performance time series, per session. Then peak (resp. trough) categories were associated 750 to trials occurring on oscillatory peaks (resp. troughs) of the considered time series of the session. All 751 following analysis concerning firing rates, LFP power and coherency and SFC estimates were compared 752 across "peak" and "trough" trials, across all channels and all sessions using non-parametric rank sum 753 tests ( $\mathrm{n}=19$ sessions, 2 monkeys).

\section{Human experiments - Human participants}

755 Thirty-five subjects participated in the study (mean age $26.5 \pm 8.35$ years, fifteen females). Among these, 756 nineteen subjects (age $25.2 \pm 2.0$, five females) were recorded with a video eye tracker (Eyelink 1000, 757 SR Research) while performing the task. All participants were totally naïve to the purpose of the 758 experiment, reported no history of neurological or psychiatric disorders, had normal or corrected-to759 normal vision and participated voluntarily after providing signed informed consent. Inclusion criteria 760 included right-handedness, which was self-reported in all participants. All individual data was 761 anonymized. All human experiments were performed in accordance with relevant guidelines and 762 regulations (the declaration of Helsinski). All experimental protocols were approved by the CNRS 
763 (research institution acting as promotor) and the CPP-Sud-Est (acting as licensing committee). The

764 project authorization is \#ID RCB 2017-A03612-51.

\section{Human experiments - Behavioral task}

766 Participants were seated in a comfortable chair in a dark room with their head positioned on a chin-rest

767 and their eyes directed towards a computer screen (24 inches), $56 \mathrm{~cm}$ away. A custom-made script using 768 Psychotoolbox(Brainard, 1997) in MATLAB (Mathworks) was used to present the stimuli and record 769 performance. Participants were asked to perform a vigilance task. At the beginning of each trial, participants were exposed to a gray-background screen with one-hundred black and white Gabor patches randomly distributed over the screen, of varying orientation, all moving toward different directions at a constant very slow velocity. After a randomized and unpredictable time period that spanned between 5 and 50 seconds, one of these gabor patches (target stimulus) started to shiver.

774 Participants' task consisted in finding this target gabor stimulus, freely exploring the screen with their eyes. Participants were instructed to point toward the target using a mouse-controlled cursor and produce a left button press, as fast as possible. The subjects' response initiated a new trial. Participants had to perform consecutive trials during 75 minutes. Importantly, subjects were instructed that there would be no rest periods. For a subset of subjects, during the task, a remote gaze tracking capture system (Eyelink 1000, SR Research) was used to continuously record eye movements, blinking and pupil size. These data were recorded at a sampling rate of $500 \mathrm{~Hz}$. The eye tracker was calibrated for each subject before the main task was initiated.

Human experiments - Behavioral performance as a function of session time and spectral analyses

783 Participants' performance was measured by studying the fluctuations of the target detection time (DT) 784 along the task. Detection times are defined as the time between the onset of the target stimulus and 785 the subject's button press. In this way, we obtained the time series of the detection times. In order to 786 study the spectral decomposition of this time series, we first equalized its sampling rate to 1000 samples 787 per hour using a least-square interpolation method, allowing a good time resolution for frequency 788 analysis. Spectral analyses were the same as those performed on the monkey behavioral data. 789 Specifically, the spectral analysis of this time series was performed on detrended data using a Morlet Wavelet transform. Standard error represented on the power spectrum is calculated along the time dimension of the wavelet analysis. We established statistical significance by reshuffling trials presentation time across the session (1000 times) and generating a random distribution of signal spectral content. Significance was then computed by comparing each session power spectrum to its corresponding 95\% confidence interval. 
bioRxiv preprint doi: https://doi.org/10.1101/2021.05.26.445730; this version posted May 26, 2021. The copyright holder for this preprint (which was not certified by peer review) is the author/funder. All rights reserved. No reuse allowed without permission.

796 Pupil size was recorded using the video eye tracking system. Blinking-related artifact was removed as 797 follows. A cubic spline interpolation method was applied to fill in data between blink onset and blink 798 offset (defined as a sharp increase and decrease in pupil size). After removing these blinking artifacts, 799 pupil size time series was down-sampled to $1 \mathrm{~Hz}$ and data was then processed using the same method 800 as the behavioral detection time series. Namely, the spectral analysis of this time series was performed 801 on detrended data using a Morlet Wavelet transform. Standard error represented on the power 802 spectrum is calculated along the time dimension of the wavelet analysis. We established statistical 803 significance by reshuffling trials presentation time across the session (1000 times) and generating a 804 random distribution of signal spectral content. Significance was then computed by comparing each 805 session power spectrum to its corresponding 95\% confidence interval. 


\section{References}

Abrahamyan, A., Silva, L.L., Dakin, S.C., Carandini, M., and Gardner, J.L. (2016). Adaptable history biases in human perceptual decisions. PNAS 113, E3548-E3557.

Allen, W.E., Chen, M.Z., Pichamoorthy, N., Tien, R.H., Pachitariu, M., Luo, L., and Deisseroth, K. (2019). Thirst regulates motivated behavior through modulation of brainwide neural population dynamics. Science 364.

Arieli, A., Sterkin, A., Grinvald, A., and Aertsen, A. (1996). Dynamics of ongoing activity: explanation of the large variability in evoked cortical responses. Science $273,1868-1871$.

Aston-Jones, G., and Cohen, J.D. (2005). An integrative theory of locus coeruleusnorepinephrine function: adaptive gain and optimal performance. Annu Rev Neurosci 28, 403-450.

Astrand, E., Wardak, C., Baraduc, P., and Ben Hamed, S. (2016). Direct Two-Dimensional Access to the Spatial Location of Covert Attention in Macaque Prefrontal Cortex. Curr. Biol. 26, 1699-1704.

Astrand, E., Wardak, C., and Ben Hamed, S. (2020). Neuronal population correlates of target selection and distractor filtering. Neurolmage 209, 116517.

B, L., S, S., and G, G. (2012). Pupillometry: A Window to the Preconscious? Perspectives on Psychological Science : A Journal of the Association for Psychological Science 7.

Bagherzadeh, Y., Baldauf, D., Pantazis, D., and Desimone, R. (2020). Alpha Synchrony and the Neurofeedback Control of Spatial Attention. Neuron 105, 577-587.e5.

Barttfeld, P., Uhrig, L., Sitt, J.D., Sigman, M., Jarraya, B., and Dehaene, S. (2015). Signature of consciousness in the dynamics of resting-state brain activity. Proc Natl Acad Sci U S A 112, 887-892.

Beaman, C.B., Eagleman, S.L., and Dragoi, V. (2017). Sensory coding accuracy and perceptual performance are improved during the desynchronized cortical state. Nature Communications 8, 1308.

Beane, M., and Marrocco, R.T. (2004). Norepinephrine and acetylcholine mediation of the components of reflexive attention: implications for attention deficit disorders. Prog Neurobiol 74, 167-181.

Beatty, J., and Lucero-Wagoner, B. (2000). The pupillary system. In Handbook of Psychophysiology, 2nd Ed, (New York, NY, US: Cambridge University Press), pp. 142-162.

Bello, F.D., Hassen, S.B.H., Astrand, E., and Hamed, S.B. (2020). Selection and suppression of visual information in the macaque prefrontal cortex. BioRxiv 2020.03.25.007922.

Bollimunta, A., Chen, Y., Schroeder, C.E., and Ding, M. (2008). Neuronal Mechanisms of Cortical Alpha Oscillations in Awake-Behaving Macaques. J. Neurosci. 28, 9976-9988. 
842 Bonnefond, A., Doignon-Camus, N., Touzalin-Chretien, P., and Dufour, A. (2010). Vigilance 843 and intrinsic maintenance of alert state: An ERP study. Behavioural Brain Research 211, 185844190.

845 Brainard, D.H. (1997). The Psychophysics Toolbox. Spat Vis 10, 433-436.

846 Busch, N.A., and VanRullen, R. (2010). Spontaneous EEG oscillations reveal periodic sampling 847 of visual attention. Proc. Natl. Acad. Sci. U.S.A. 107, 16048-16053.

848 Busch, N.A., Dubois, J., and VanRullen, R. (2009). The phase of ongoing EEG oscillations 849 predicts visual perception. J. Neurosci. 29, 7869-7876.

850 Buschman, T.J., and Miller, E.K. (2007a). Top-down versus bottom-up control of attention in 851 the prefrontal and posterior parietal cortices. Science 315, 1860-1862.

852 Buschman, T.J., and Miller, E.K. (2007b). Top-down versus bottom-up control of attention in 853 the prefrontal and posterior parietal cortices. Science 315, 1860-1862.

854 Buschman, T.J., and Miller, E.K. (2009). Serial, Covert, Shifts of Attention during Visual Search 855 are Reflected by the Frontal Eye Fields and Correlated with Population Oscillations. Neuron 856 63, 386-396.

857 Chalk, M., Herrero, J.L., Gieselmann, M.A., Delicato, L.S., Gotthardt, S., and Thiele, A. (2010). 858 Attention Reduces Stimulus-Driven Gamma Frequency Oscillations and Spike Field 859 Coherence in V1. Neuron 66, 114-125.

860 Chan, R.W., Leong, A.T.L., Ho, L.C., Gao, P.P., Wong, E.C., Dong, C.M., Wang, X., He, J., Chan, 861 Y.-S., Lim, L.W., et al. (2017). Low-frequency hippocampal-cortical activity drives brain-wide 862 resting-state functional MRI connectivity. Proc Natl Acad Sci U S A 114, E6972-E6981.

863 Compton, R.J., Gearinger, D., and Wild, H. (2019). The wandering mind oscillates: EEG alpha 864 power is enhanced during moments of mind-wandering. Cogn Affect Behav Neurosci 19, $865 \quad 1184-1191$.

866 Cowley, B.R., Snyder, A.C., Acar, K., Williamson, R.C., Yu, B.M., and Smith, M.A. (2020). Slow 867 Drift of Neural Activity as a Signature of Impulsivity in Macaque Visual and Prefrontal Cortex. 868 Neuron 108, 551-567.e8.

869 De Sousa, C., Gaillard, C., Di Bello, C., Ben Hadj Hassen, F., and Ben Hamed, S. (2021).

870 Behavioral validation of novel high resolution attention decoding method from multi-units \& 871 local field potentials. Neurolmage 231, 117853.

872 Demertzi, A., Tagliazucchi, E., Dehaene, S., Deco, G., Barttfeld, P., Raimondo, F., Martial, C., 873 Fernández-Espejo, D., Rohaut, B., Voss, H.U., et al. (2019). Human consciousness is 874 supported by dynamic complex patterns of brain signal coordination. Science Advances 5, 875 eaat7603.

876 van Dijk, H., Schoffelen, J.-M., Oostenveld, R., and Jensen, O. (2008). Prestimulus oscillatory 877 activity in the alpha band predicts visual discrimination ability. J Neurosci $28,1816-1823$. 
878 Dragone, A., Lasaponara, S., Pinto, M., Rotondaro, F., De Luca, M., and Doricchi, F. (2018).

879 Expectancy modulates pupil size during endogenous orienting of spatial attention. Cortex $880 \quad 102,57-66$.

881 Dugué, L., and VanRullen, R. (2017). Transcranial Magnetic Stimulation Reveals Intrinsic

882 Perceptual and Attentional Rhythms. Front Neurosci 11.

883 Dugué, L., Marque, P., and VanRullen, R. (2014). Theta Oscillations Modulate Attentional

884 Search Performance Periodically. Journal of Cognitive Neuroscience 27, 1-14.

885 Dugué, L., Roberts, M., and Carrasco, M. (2016). Attention Reorients Periodically. Curr Biol $88626,1595-1601$.

887 Ebitz, R.B., and Moore, T. (2017). Selective Modulation of the Pupil Light Reflex by

888 Microstimulation of Prefrontal Cortex. J. Neurosci. 37, 5008-5018.

889 Ekstrom, L.B., Roelfsema, P.R., Arsenault, J.T., Bonmassar, G., and Vanduffel, W. (2008).

890 Bottom-up dependent gating of frontal signals in early visual cortex. Science 321, 414-417.

891 Eldar, E., Cohen, J.D., and Niv, Y. (2013). The effects of neural gain on attention and learning. 892 Nat Neurosci 16, 1146-1153.

893 Engel, T.A., Steinmetz, N.A., Gieselmann, M.A., Thiele, A., Moore, T., and Boahen, K. (2016).

894 Selective modulation of cortical state during spatial attention. Science 354, 1140-1144.

895 Everitt, B.J., and Robbins, T.W. (1997). Central cholinergic systems and cognition. Annu Rev 896 Psychol 48, 649-684.

897 Fiebelkorn, I.C., Pinsk, M.A., and Kastner, S. (2018a). A Dynamic Interplay within the 898 Frontoparietal Network Underlies Rhythmic Spatial Attention. Neuron 99, 842-853.e8.

899 Fiebelkorn, I.C., Pinsk, M.A., and Kastner, S. (2018b). A Dynamic Interplay within the 900 Frontoparietal Network Underlies Rhythmic Spatial Attention. Neuron 99, 842-853.e8.

901 Fiebelkorn, I.C., Pinsk, M.A., and Kastner, S. (2019). The mediodorsal pulvinar coordinates 902 the macaque fronto-parietal network during rhythmic spatial attention. Nature 903 Communications 10, 215.

904 Fries, P. (2005). A mechanism for cognitive dynamics: neuronal communication through 905 neuronal coherence. Trends in Cognitive Sciences 9, 474-480.

906 Fries, P. (2015a). Rhythms For Cognition: Communication Through Coherence. Neuron 88, $907 \quad 220-235$.

908 Fries, P. (2015b). Rhythms For Cognition: Communication Through Coherence. Neuron 88, $909 \quad 220-235$.

910 Fries, P., Reynolds, J.H., Rorie, A.E., and Desimone, R. (2001). Modulation of oscillatory 911 neuronal synchronization by selective visual attention. Science 291, 1560-1563. 

Stimulation and Selective Visual Attention on Rhythmic Neuronal Synchronization in Macaque Area V4. J Neurosci 28, 4823-4835.

915

916

917

918

919

920

921

922

923

924

925

926

927

928

929

930

931

932

933

934

935

936

937

938

939

940

941

942

943

944

945

946

947

Gaillard, C., Ben Hadj Hassen, S., Di Bello, F., Bihan-Poudec, Y., VanRullen, R., and Ben Hamed, S. (2020a). Prefrontal attentional saccades explore space rhythmically. Nature Communications 11, 925.

Gaillard, C., Ben Hadj Hassen, S., Di Bello, F., Bihan-Poudec, Y., VanRullen, R., and Ben Hamed, S. (2020b). Prefrontal attentional saccades explore space rhythmically. Nat Commun $11,925$.

Gregoriou, G.G., Gotts, S.J., Zhou, H., and Desimone, R. (2009a). High-frequency, long-range coupling between prefrontal and visual cortex during attention. Science 324, 1207-1210.

Gregoriou, G.G., Gotts, S.J., Zhou, H., and Desimone, R. (2009b). High-frequency, long-range coupling between prefrontal and visual cortex during attention. Science 324, 1207-1210.

Grinsted, A., Moore, J.C., and Jevrejeva, S. (2004). Application of the cross wavelet transform and wavelet coherence to geophysical time series. Nonlinear Processes in Geophysics 11, 561-566.

Guo, Z., Chen, R., Zhang, K., Pan, Y., and Wu, J. (2016). The Impairing Effect of Mental Fatigue on Visual Sustained Attention under Monotonous Multi-Object Visual Attention Task in Long Durations: An Event-Related Potential Based Study. PLOS ONE 11, e0163360.

Gutnisky, D.A., Beaman, C., Lew, S.E., and Dragoi, V. (2017). Cortical response states for enhanced sensory discrimination. Elife 6.

Hassen, S.B.H., Wardak, C., and Hamed, S.B. (2019). Rhythmic variations in prefrontal interneuronal correlations, their underlying mechanisms and their behavioral correlates. BioRxiv 784850 .

Horschig, J.M., Oosterheert, W., Oostenveld, R., and Jensen, O. (2015). Modulation of Posterior Alpha Activity by Spatial Attention Allows for Controlling A Continuous BrainComputer Interface. Brain Topogr 28, 852-864.

Huang, N., and Elhilali, M. Push-pull competition between bottom-up and top-down auditory attention to natural soundscapes. ELife 9.

Huang, Z., Zhang, J., Wu, J., Mashour, G.A., and Hudetz, A.G. (2020). Temporal circuit of macroscale dynamic brain activity supports human consciousness. Sci Adv 6, eaaz0087.

van der Hulst, M. (2003). Long workhours and health. Scand J Work Environ Health 29, 171188.

Ibos, G., Duhamel, J.-R., and Hamed, S.B. (2013). A Functional Hierarchy within the Parietofrontal Network in Stimulus Selection and Attention Control. J. Neurosci. 33, 83598369. 
Jasper, A.I., Tanabe, S., and Kohn, A. (2019). Predicting Perceptual Decisions Using Visual Cortical Population Responses and Choice History. J. Neurosci. 39, 6714-6727.

Jia, X., and Kohn, A. (2011). Gamma Rhythms in the Brain. PLoS Biol 9.

951

952

953

954

955

956

957

958

959

960

961

962

963

964

965

966

967

968

969

970

971

972

973

974

975

976

977

978

979

980

981

982

Johnson, J.V., and Lipscomb, J. (2006). Long working hours, occupational health and the changing nature of work organization. Am J Ind Med 49, 921-929.

Joshi, S., Li, Y., Kalwani, R.M., and Gold, J.I. (2016). Relationships between Pupil Diameter and Neuronal Activity in the Locus Coeruleus, Colliculi, and Cingulate Cortex. Neuron 89, 221-234.

Just, M.A., Carpenter, P.A., and Miyake, A. (2003). Neuroindices of cognitive workload: Neuroimaging, pupillometric and event-related potential studies of brain work. Theoretical Issues in Ergonomics Science 4, 56-88.

Kembro, J.M., Lihoreau, M., Garriga, J., Raposo, E.P., and Bartumeus, F. (2019). Bumblebees learn foraging routes through exploitation-exploration cycles. J R Soc Interface 16, 20190103.

Kim, S.-Y., and Lim, W. (2013). Sparsely-synchronized brain rhythm in a small-world neural network. Journal of the Korean Physical Society 63, 104-113.

Klein, R., and Armitage, R. (1979). Rhythms in human performance: 1 1/2-hour oscillations in cognitive style. Science 204, 1326-1328.

Knyazev, G.G., Slobodskoj-Plusnin, J.Y., Bocharov, A.V., and Pylkova, L.V. (2011). The default mode network and EEG $\alpha$ oscillations: an independent component analysis. Brain Res 1402, 67-79.

Kramer, A. (1990). Physiological metrics of mental workload: A review of recent progress.

Leopold, D.A., and Logothetis, N.K. (1998). Microsaccades differentially modulate neural activity in the striate and extrastriate visual cortex. Exp Brain Res 123, 341-345.

Li, J.M., Bentley, W.J., Snyder, A.Z., Raichle, M.E., and Snyder, L.H. (2015). Functional connectivity arises from a slow rhythmic mechanism. Proc Natl Acad Sci U S A 112, E25272535.

Liu, Y., and Tanaka, H. (2002). Overtime work, insufficient sleep, and risk of non-fatal acute myocardial infarction in Japanese men. Occup Environ Med 59, 447-451.

Liu, Y., Rodenkirch, C., Moskowitz, N., Schriver, B., and Wang, Q. (2017). Dynamic Lateralization of Pupil Dilation Evoked by Locus Coeruleus Activation Results from Sympathetic, Not Parasympathetic, Contributions. Cell Rep 20, 3099-3112.

Lockley, S., Cronin, J., Flynn-Evans, E., Cade, B., Lee, C., Landrigan, C., Rothschild, J., Katz, J., Lilly, C., Stone, P., et al. (2004). Effect of Reducing Interns' Weekly Work Hours on Sleep and Attentional Failures. The New England Journal of Medicine 351, 1829-1837. 
983

984

985

986

987

988

989

990

991

992

993

994

995

996

997

998

999

1000

1001

1002

1003

1004

1005

1006

1007

1008

1009

1010

1011

1012

1013

1014

1015

1016

1017

Marcora, S.M., Staiano, W., and Manning, V. (2009). Mental fatigue impairs physical performance in humans. J Appl Physiol (1985) 106, 857-864.

McCormick, D.A. (1992). Neurotransmitter actions in the thalamus and cerebral cortex and their role in neuromodulation of thalamocortical activity. Progress in Neurobiology 39, 337388.

McGinley, M.J., Vinck, M., Reimer, J., Batista-Brito, R., Zagha, E., Cadwell, C.R., Tolias, A.S., Cardin, J.A., and McCormick, D.A. (2015). Waking State: Rapid Variations Modulate Neural and Behavioral Responses. Neuron 87, 1143-1161.

Mi, Y., Liao, X., Huang, X., Zhang, L., Gu, W., Hu, G., and Wu, S. (2013). Long-period rhythmic synchronous firing in a scale-free network. Proc Natl Acad Sci U S A 110, E4931-4936.

Milton, R., Shahidi, N., and Dragoi, V. (2020). Dynamic states of population activity in prefrontal cortical networks of freely-moving macaque. Nature Communications 11, 1948.

Mo, J., Schroeder, C.E., and Ding, M. (2011). Attentional Modulation of Alpha Oscillations in Macaque Inferotemporal Cortex. J Neurosci 31, 878-882.

Mo, J., Liu, Y., Huang, H., and Ding, M. (2013). Coupling between visual alpha oscillations and default mode activity. Neuroimage $68,112-118$.

Moore, T., and Zirnsak, M. (2017). Neural Mechanisms of Selective Visual Attention. Annu Rev Psychol 68, 47-72.

Nassar, M.R., Rumsey, K.M., Wilson, R.C., Parikh, K., Heasly, B., and Gold, J.I. (2012). Rational regulation of learning dynamics by pupil-linked arousal systems. Nat Neurosci 15, 10401046.

Oby, E.R., Golub, M.D., Hennig, J.A., Degenhart, A.D., Tyler-Kabara, E.C., Yu, B.M., Chase, S.M., and Batista, A.P. (2019). New neural activity patterns emerge with long-term learning. Proc Natl Acad Sci U S A 116, 15210-15215.

Okun, M., Steinmetz, N.A., Cossell, L., lacaruso, M.F., Ko, H., Barthó, P., Moore, T., Hofer, S.B., Mrsic-Flogel, T.D., Carandini, M., et al. (2015). Diverse coupling of neurons to populations in sensory cortex. Nature $521,511-515$.

Okun, M., Steinmetz, N.A., Lak, A., Dervinis, M., and Harris, K.D. (2019). Distinct Structure of Cortical Population Activity on Fast and Infraslow Timescales. Cerebral Cortex 29, 21962210.

Oostenveld, R., Fries, P., Maris, E., and Schoffelen, J.-M. (2011). FieldTrip: Open source software for advanced analysis of MEG, EEG, and invasive electrophysiological data. Comput Intell Neurosci 2011, 156869.

Palva, J.M., and Palva, S. (2011). Roles of multiscale brain activity fluctuations in shaping the variability and dynamics of psychophysical performance. Prog Brain Res 193, 335-350. 
1018 Paneri, S., and Gregoriou, G.G. (2017). Top-Down Control of Visual Attention by the

Parto Dezfouli, M., Khamechian, M.B., Treue, S., Esghaei, M., and Daliri, M.R. (2018). Neural Neurosci 12.

1023 Pisupati, S., Chartarifsky-Lynn, L., Khanal, A., and Churchland, A.K. Lapses in perceptual decisions reflect exploration. ELife 10.

1025 Posner, M.I. (2016). Orienting of attention: Then and now. Q J Exp Psychol (Hove) 69, 18641875.

Proctor, S.P., White, R.F., Robins, T.G., Echeverria, D., and Rocskay, A.Z. (1996). Effect of overtime work on cognitive function in automotive workers. Scand J Work Environ Health $22,124-132$.

1030 Rabinowitz, N.C., Goris, R.L., Cohen, M., and Simoncelli, E.P. (2015). Attention stabilizes the shared gain of V4 populations. ELife 4, e08998.

1032

1033

1034

1035

Reimer, J., Froudarakis, E., Cadwell, C.R., Yatsenko, D., Denfield, G.H., and Tolias, A.S. (2014). Pupil fluctuations track fast switching of cortical states during quiet wakefulness. Neuron 84 , 355-362.

Reynaud, A.J., Froesel, M., Guedj, C., Ben Hadj Hassen, S., Cléry, J., Meunier, M., Ben Hamed, S., and Hadj-Bouziane, F. (2019). Atomoxetine improves attentional orienting in a predictive context. Neuropharmacology 150, 59-69.

Ros, T., Michela, A., Bellman, A., Vuadens, P., Saj, A., and Vuilleumier, P. (2017). Increased Alpha-Rhythm Dynamic Range Promotes Recovery from Visuospatial Neglect: A Neurofeedback Study. Neural Plast 2017.

Saj, A., Ros, T., Michela, A., and Vuilleumier, P. (2018). Effect of a single early EEG neurofeedback training on remediation of spatial neglect in the acute phase. Ann Phys Rehabil Med 61, 111-112.

Sekine, M., Chandola, T., Martikainen, P., McGeoghegan, D., Marmot, M., and Kagamimori, S. (2006). Explaining social inequalities in health by sleep: the Japanese civil servants study. Journal of Public Health 28, 63-70.

Shields, M. (1999). Long working hours and health. Health Rep 11, 33-48(Eng); 37-55(Fre).

Siegel, M., Donner, T.H., Oostenveld, R., Fries, P., and Engel, A.K. (2008). Neuronal synchronization along the dorsal visual pathway reflects the focus of spatial attention. Neuron 60, 709-719.

Sirois, S., and Brisson, J. (2014). Pupillometry. WIREs Cognitive Science 5, 679-692.

Smallwood, J., and Schooler, J.W. (2015). The science of mind wandering: empirically navigating the stream of consciousness. Annu Rev Psychol 66, 487-518. 
1054

1055

1056

1057

1058

1059

1060

1061

1062

1063

1064

1065

1066

1067

1068

1069

1070

1071

1072

1073

1074

1075

1076

1077

1078

1079

1080

1081

1082

1083

1084

1085

1086

1087

Snyder, A.C., Morais, M.J., and Smith, M.A. (2016). Dynamics of excitatory and inhibitory networks are differentially altered by selective attention. J Neurophysiol 116, 1807-1820.

Sokejima, S., and Kagamimori, S. (1998). Working hours as a risk factor for acute myocardial infarction in Japan: case-control study. BMJ 317, 775-780.

Steinmetz, N.A., and Moore, T. (2014). Eye movement preparation modulates neuronal responses in area V4 when dissociated from attentional demands. Neuron 83, 496-506.

Stringer, C., Pachitariu, M., Steinmetz, N., Carandini, M., and Harris, K.D. (2019). Highdimensional geometry of population responses in visual cortex. Nature 571, 361-365.

Thomson, D.R., Besner, D., and Smilek, D. (2015). A resource-control account of sustained attention: evidence from mind-wandering and vigilance paradigms. Perspect Psychol Sci 10, 82-96.

Tremblay, S., Doucet, G., Pieper, F., Sachs, A., and Martinez-Trujillo, J. (2015). Single-Trial Decoding of Visual Attention from Local Field Potentials in the Primate Lateral Prefrontal Cortex Is Frequency-Dependent. J. Neurosci. 35, 9038-9049.

Urai, A.E., de Gee, J.W., Tsetsos, K., and Donner, T.H. (2019). Choice history biases subsequent evidence accumulation. ELife 8, e46331.

VanRullen, R. (2018). Attention Cycles. Neuron 99, 632-634.

Vernet, M., Stengel, C., Quentin, R., Amengual, J.L., and Valero-Cabré, A. (2019).

Entrainment of local synchrony reveals a causal role for high-beta right frontal oscillations in human visual consciousness. Sci Rep 9, 14510.

Vinck, M., van Wingerden, M., Womelsdorf, T., Fries, P., and Pennartz, C.M.A. (2010). The pairwise phase consistency: A bias-free measure of rhythmic neuronal synchronization. Neurolmage 51, 112-122.

Vinck, M., Batista-Brito, R., Knoblich, U., and Cardin, J.A. (2015). Arousal and locomotion make distinct contributions to cortical activity patterns and visual encoding. Neuron 86, 740-754.

Virtanen, M., Singh-Manoux, A., Ferrie, J.E., Gimeno, D., Marmot, M.G., Elovainio, M., Jokela, M., Vahtera, J., and Kivimäki, M. (2009). Long Working Hours and Cognitive Function. Am J Epidemiol 169, 596-605.

van Vugt, B., Dagnino, B., Vartak, D., Safaai, H., Panzeri, S., Dehaene, S., and Roelfsema, P.R. (2018). The threshold for conscious report: Signal loss and response bias in visual and frontal cortex. Science 360, 537-542.

Warm, J.S., Parasuraman, R., and Matthews, G. (2008). Vigilance Requires Hard Mental Work and Is Stressful. Hum Factors 50, 433-441. 
Wasmuht, D.F., Parker, A.J., and Krug, K. (2019). Interneuronal correlations at longer time scales predict decision signals for bistable structure-from-motion perception. Scientific Reports 9, 11449.

1091

1092

1093

1094

1095

1096

1097

1098

1099

1100

1101

1102

1103

1104

1105

1106

1107

1108

1109

1110

1111

1112

1113

1114

1115

1116

1117

1118

Weissman, D.H., Roberts, K.C., Visscher, K.M., and Woldorff, M.G. (2006). The neural bases of momentary lapses in attention. Nat Neurosci 9, 971-978.

van der Wel, P., and van Steenbergen, H. (2018). Pupil dilation as an index of effort in cognitive control tasks: A review. Psychon Bull Rev 25, 2005-2015.

Yüzgeç, Ö., Prsa, M., Zimmermann, R., and Huber, D. (2018). Pupil Size Coupling to Cortical States Protects the Stability of Deep Sleep via Parasympathetic Modulation. Curr Biol 28, 392-400.e3.

Zareian, B., Daliri, M.R., Maboudi, K., Moghaddam, H.A., Treue, S., and Esghaei, M. (2018). Attention enhances LFP phase coherence in macaque visual cortex, improving sensory processing. BioRxiv 499756.

Zareian, B., Maboudi, K., Daliri, M.R., Abrishami Moghaddam, H., Treue, S., and Esghaei, M. (2020). Attention strengthens across-trial pre-stimulus phase coherence in visual cortex, enhancing stimulus processing. Sci Rep 10.

Pupil Dilation Reflects the Creation and Retrieval of Memories - Stephen D. Goldinger, Megan H. Papesh, 2012. 


\section{Supplementary figures}

1120

a

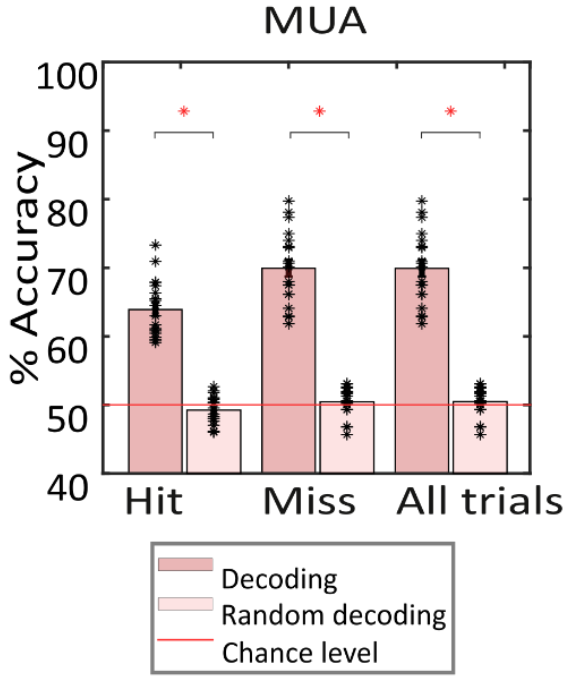

b

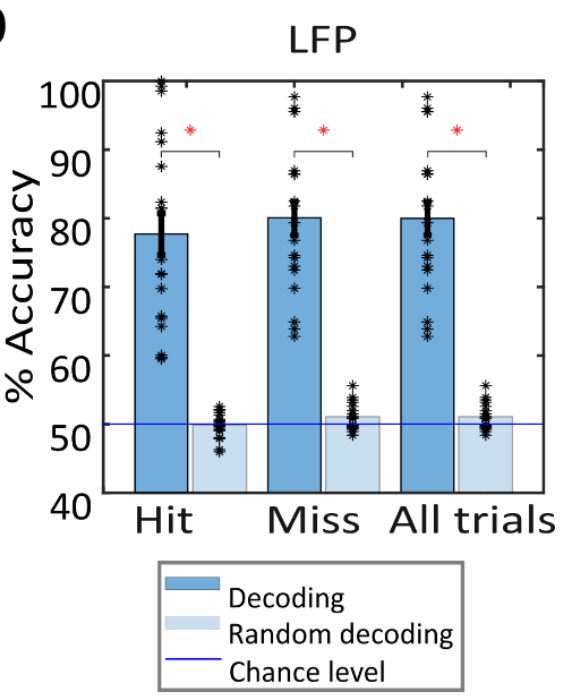

1122

1123

1124

1125

1126

1127

1128

1129

1130

1131

1132

1133

1134

1135

1136

1137

1138

1139

Figure S1: Hit vs. Miss decoding accuracies from prefrontal MUA and LFP signals. (A) Decoding accuracies for Hit, Miss or all trials (Hit and Miss) classification from prefrontal MUA presented in pink compare to a random decoding presented in white $(n=19$; ranksum test - *p value $<0.0001)$. (B) Decoding accuracies for Hit, Miss or both trials (Hit and Miss) classification from prefrontal LFP frequency content presented in blue compare to a random decoding presented in white $(n=19$; ranksum test $-{ }^{*} p$ value $\left.<0.0001\right)$. 
a

\section{Freq. (cycle/hour)}

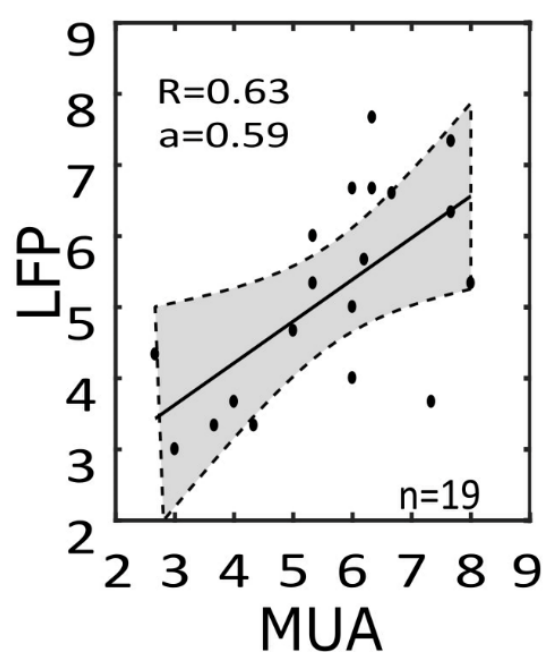

b

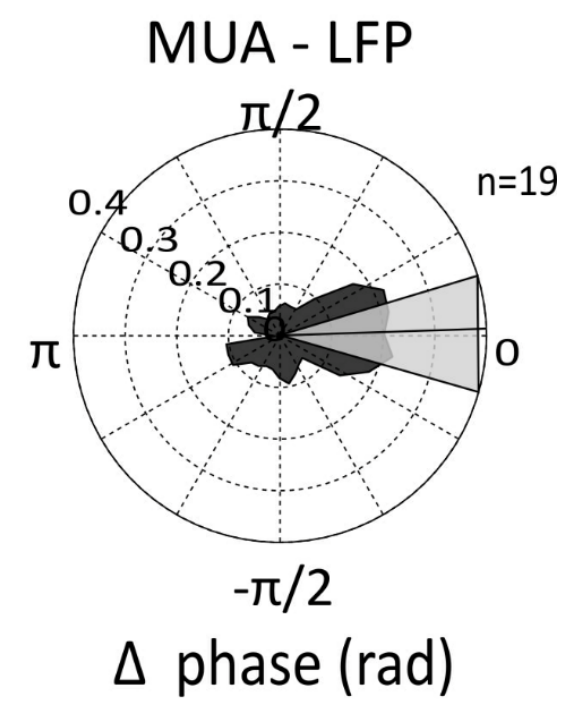

1142

1143

1144

1145

1146

1147

1148

1149

1150

1151

1152

1153

1154

1155

1156

1157

Figure S2: Ultra-slow rhythmic fluctuations in accuracies at target decoding on hit vs. miss trials from prefrontal MUA and LFP signals. (A) Correlation between ultra-slow oscillations identified in LFP and MUA hit vs. miss decoding accuracies (Spearman correlation, $\mathrm{p}<0.01$ ) and corresponding phase difference (B) (circular Kuiper's test $p<0.05)$. 
a

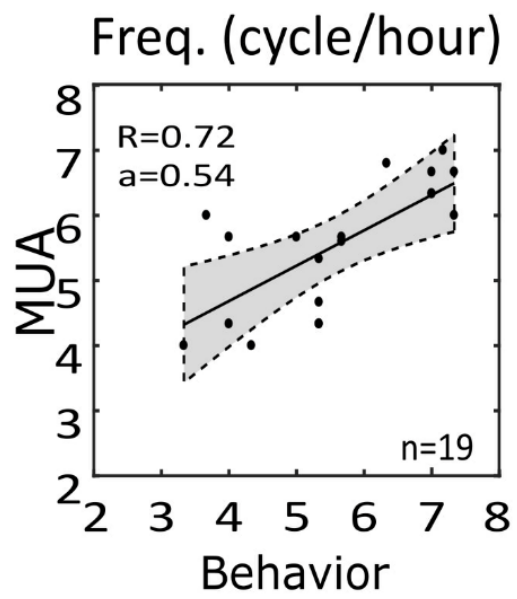

b

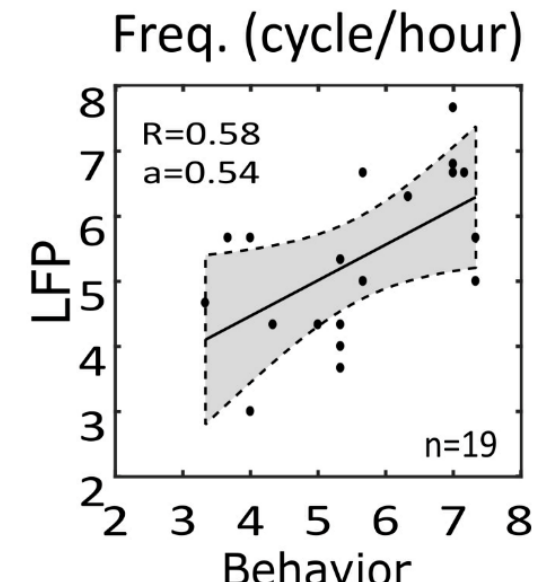

1162

Figure S3: Ultra-slow rhythmic fluctuations in accuracies at decoding attention-related information

from prefrontal MUA and LFP signals. (A) Correlation between ultra-slow oscillations identified in MUA spatial decoding accuracies and in behavioral performance (Spearman correlation, $p<0.01$ ) and corresponding phase difference (B) (circular Kuiper's test $p<0.05$ ). 


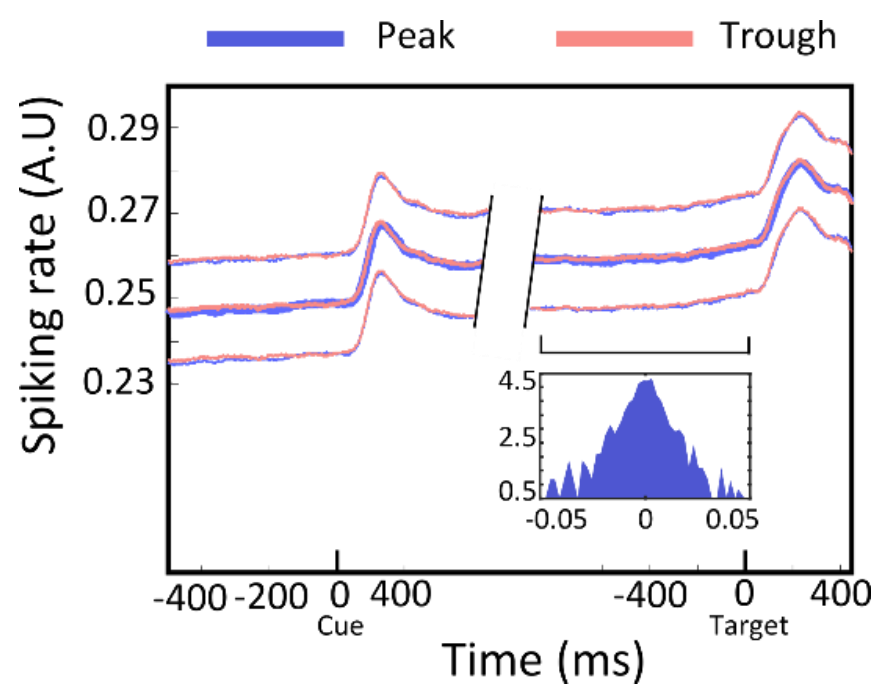

Figure S4: Ultra-slow rhythm does not impact averaged FEF MUA response to Cue and Target 